\title{
Theoretical and experimental description of permeability of peptide-containing membranes
}

\author{
Dissertation \\ zur Erlangung des Doktorgrades \\ der Mathematisch-Naturwissenschaftlichen Fakultäten \\ der Georg-August-Universität zu Göttingen
}

vorgelegt von

Ivan Makarov

aus Kiew, Ukraine

Göttingen 2005 
D7

Referent : Prof. Tim Salditt

Korefferent : Prof. Thomas Heimburg

Tag der mündlichen Prüfung : 23.2.2005 
Für meine $\mathfrak{M u t t e r}$ 
Freund, ef ift aud) genug. İm f̧all bu mehr millift lejen,

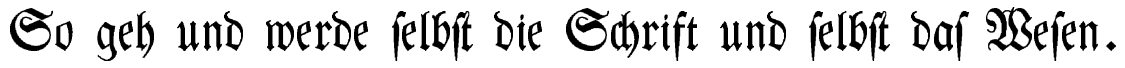

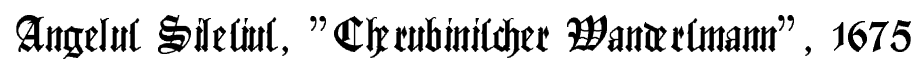




\section{Danksagung}

Hier will ich mich ganz herzlich bei allen bedanken, die mich bei der Erstellung dieses Werkes unterstützt haben:

Prof. Thomas Heimburg, für die gute und freundliche Betreuung und für interessante Diskussionen;

Prof. Tim Salditt,

für die Unterstützung dieser Arbeit von Seiten der Universität Göttingen;

Prof. Detlev Schild,

für die Unterstützung dieser Arbeit von Seiten des Graduierten Kollegs GRK 723;

Heiko Seeger,

für die freundliche Hilfe bei der Korrektur der deutschen und englischen Sprache und seiner freundlichen Beratung bei bürokratischen Fragen;

\section{Dr. Fabiana Ganz,}

für die Diskussionen und die Einführung in die Chromatographie;

Denis Pollakowski,

für die angenehmen Gespräche über Klassische Musik;

Mattias Fidorra,

für die nette Hilfe beim Umzug;

Jan Plotzki,

für die angenehme Hilfe bei technischen Problemen;

Martin Fechner,

für die freundliche Hilfe bei Computerproblemen;

Nicht zuletzt bedanke ich mich bei den Mitarbeitern der Werkstätten des MPI für biophysikalische Chemie, Göttingen und des Niels Bohr Instituts, Kopenhagen. 


\section{Contents}

$\begin{array}{llr}1 & \text { Introduction } & 8\end{array}$

1.1 Historical introduction into membrane permeability studies . . 8

1.2 Membrane structure and properties . . . . . . . . . . . . . 12

1.3 Membrane permeability : general view ......... 16

1.4 Permeability models and simulation approaches . . . . . . . 21

1.5 Phase transition, local fluctuations and permeability . . . . 22

2 Experimental Methods $\quad 28$

2.1 Differential Scanning Calorimetry . . . . . . . . . . . 28

2.2 Probe preparation for Differential Scanning Calorimetry . . 29

2.3 Fluorescence Correlation spectroscopy . . . . . . . . . . . . 31

2.4 Probe preparation for Fluorescence Correlation spectroscopy • 42

$\begin{array}{lll}3 & \text { Simulation approach } & 44\end{array}$

3.1 Ising-like model on a hexagonal lattice . . . . . . . . . . . 44

3.2 Heat capacity calculations and Glauber algorhitm . . . . . . . 46 
3.3 Peptides . . . . . . . . . . . . . . . . . . . . . . . . 48

3.4 Introduction of compressibility into the model and permeability simulation 50

4 Results

56

4.1 Gramicidin A aggregation study : calorimetry results . . . . . 56

4.2 Gramicidin A aggregation study : Monte Carlo results . . . . 57

4.3 Gramicidin A aggregation study : Local Fluctuations . . . . . 60

4.4 Membrane permeability : FCS results ........... 63

4.5 Membrane permeability : Monte Carlo results . . . . . . . 66

4.6 Discussion and perspectives . . . . . . . . . . 72

A Chromatography procedure 


\section{Chapter 1}

\section{Introduction}

\subsection{Historical introduction into membrane per- meability studies}

Membranes play the role of barriers, which separates inside and outside of the cell. They allow substances like water, oxygen or carbon dioxide, which need to be transported fast in and out of the cell, to move easily through the membrane. However they need to make permeation of sodium, potassium, calcium and protons difficult in order to maintain the ion gradients which are necessary for biological function. They contain channel proteins and ion pumps to produce such gradients as well.

In the 1855-1877 the existence of an osmotic barrier between the protoplasm of plant cells and their environment, was shown experimentally (Nägeli and Cramer, 1855), (Vries de, 1871), (Vries de, 1884), (Pfeffer, 1877). This was clear from observations of protoplasmic permeability. Plant cells protoplasts were permeable to water, but not to many different solutes, like sucrose (Vries de, 1871).

In these experiments the retraction of the protoplasm from the cellulose wall 


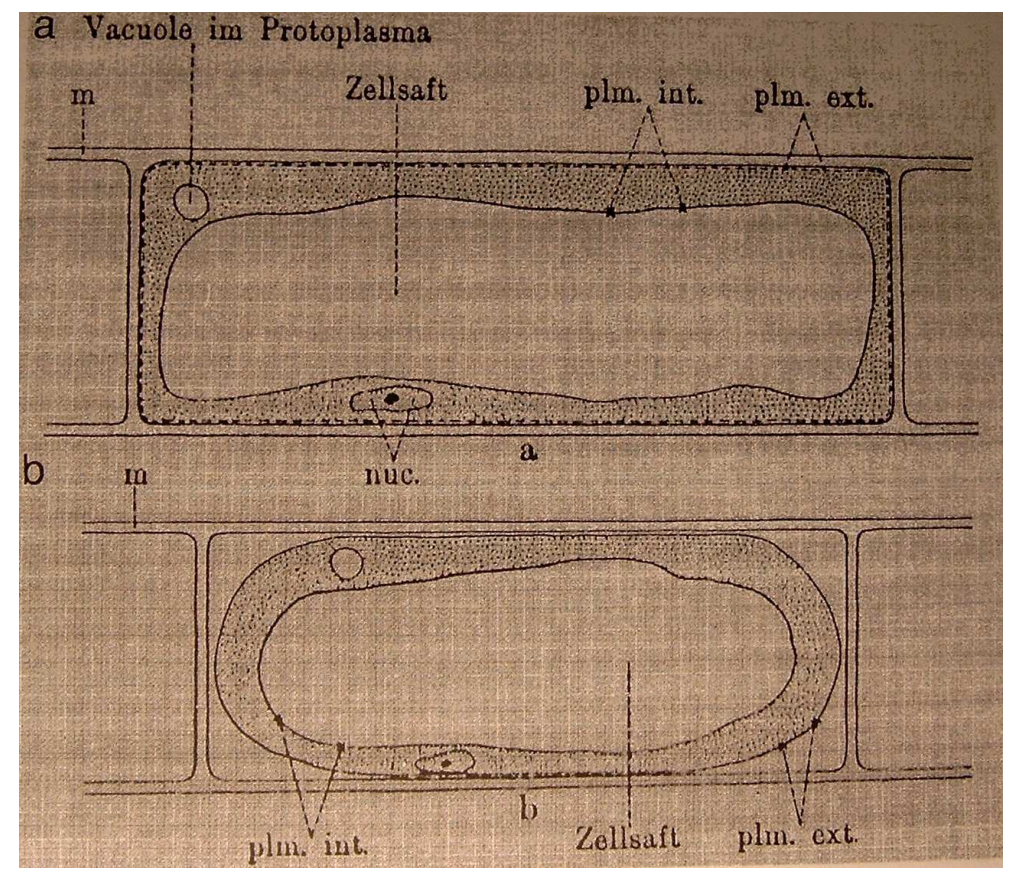

Figure 1.1: Plant cell from an osmotic point of view. a)-normal state. b) -plasmolysed state,( Picture taken from (Kleinzeller, 1999)) .

of the plant cell was observed, when the cell was exposed to solutes, for which the membrane was not permeable. ( fig. 1.1 )

Overton refined this method, using the law, that the osmotic pressure of a solution is equal to the sum of partial osmotic pressures of the components. This simplified the experiments, decreased the amounts of solutes used, and, therefore resulted in decrease in a toxicity of the experiments.

After van't Hoff's demonstrated the analogy between gas laws and the osmotic properties of solutes in 1887 , Overton illustrated the permeation experiments (Overton, 1895) using comparison between a plant cell with a soccer ball, exposed to outside pressure changes. ( fig. 1.2 ) The leather cover of the balloon is permeable to all gases, but the internal rubber is not. Increasing of the external pressure would result in shrinkage of the rubber. 
But if the rubber is permeable to the gas pressure of this gas inside and outside the ball would be the same, therefore changes in pressure would cause no effect on the rubber.

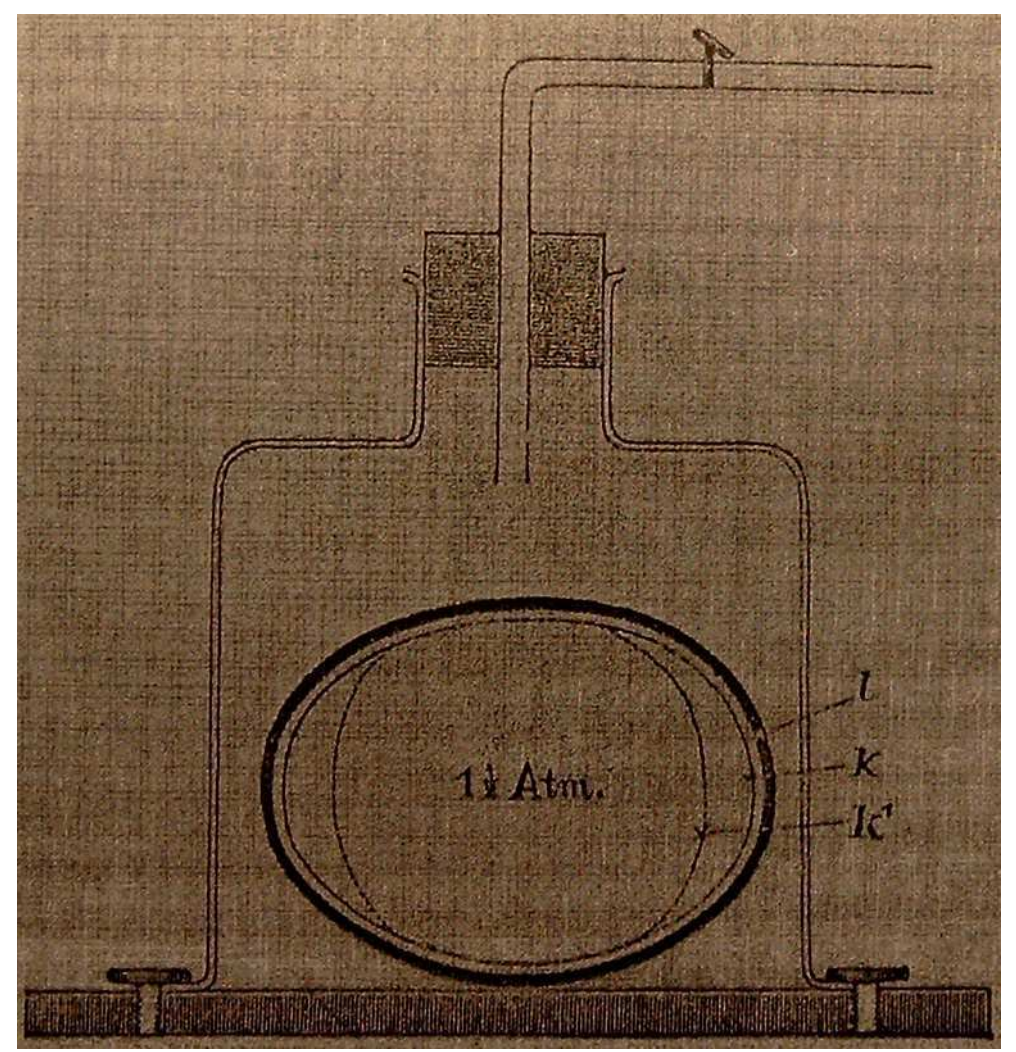

Figure 1.2: Illustration of the analogy between plant cell and soccer ball, exposed to outside pressure changes. The leather cover of the balloon is permeable to all gases, but the internal rubber is not. Increasing of the external pressure would result in shrinkage of the rubber. But if the rubber is permeable to the gas pressure of this gas inside and outside the ball would be the same, therefore changes in pressure would cause no effect on the rubber, (Picture taken from (Kleinzeller, 1999)) .

The structure of membrane was not known at that time, as well the material it made of. Scientists have thought about of an oil-made membrane, or membrane, which consists of proteins, especially after Ramsden(Ramsden, 1904) demonstration of solid film formation at the contact surface of a protein 
solution and air.

Quincke (Quincke, 1888) suggested the existence of a thin lipid layer at the boundary of the protoplasm.

Pfeffer after his experiments with dye uptake (Pfeffer, 1886) suggested, that the cell membrane not only demonstrates passive barriers to different solutes, but shows capability to take up or exclude solutes. His hypothesis, that a membrane is "protoplasmic organ, capable of regulating exchange of solutes" was outstanding for his time.

He suggested as well, that the main feature, which determines the permeability of a solute, is its solubility in the membrane. This idea was adopted by Overton, after hundreds of experiments with different solutes. From experiments of Newson (Newson, 1773), Hamburger (Hamburger, 1887) and Overton (Overton, 1895), (Overton, 1899) it was clear, that this suggested lipid-impregnated boundary was a common feature of plant and animal cells. Overton (Overton, 1899) suggested, that lipid material of this boundary can have properties similar to cholesterol esters and phospholipids, because of their capability of immiscibility in water and possibility of wetting cells with aqueous solute solution.

Overton (Overton, 1899), (Overton, 1855) and Meyer (Meyer, 1899) developed independently the lipid theory of narcosis. They suggested, that narcotic action on nervous tissue was based on high solubility of narcotics in lipids. They discovered the role of narcotic partition between lipid part and cellular water and showed a correlation between the effect of narcotic and his lipid : water partition. Overton developed a practical method to determine the minimal concentration of narcotic, needed for complete narcosis. This was important for clinical use. 
He observed that narcotics passed fast through the cell boundary layer and may affect its osmotic properties or intracellular components. The exact mechanism of narcotic action was still an open question.

Overton's concepts of membrane permeability allowed him to describe the permeability properties of a surprisingly wide range of solutes, without knowing the structure of the lipid membrane.

\subsection{Membrane structure and properties}

The membrane structure is a lipid bilayer ( fig. 1.3 ), wich consists of two layers of lipids ( fig. 1.4 ), with hydrophilic heads exposed to an aqueous solution outside, and hydrophobic tails, oriented inside the bilayer.

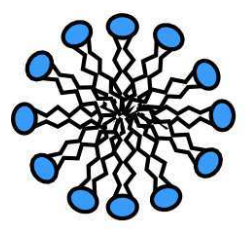

a) micelle

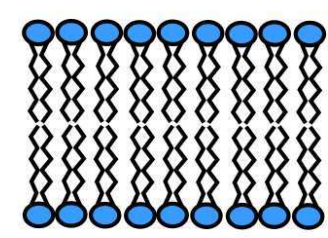

b) bilayer

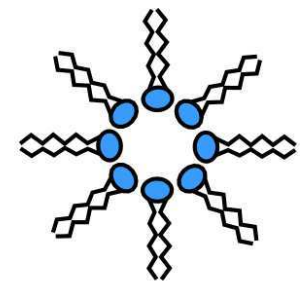

c) inverse micelle

Figure 1.3: Lipid bilayer b)and nonlameller phases micelle a) and inverse micelle b).

The presence of a bilayer at the cell boundary was shown in the work of Danielli and Dawson (Danielli and J., 1935), who observed, that the amount of lipids, that was extracted from red blood cells is sufficient to cover the cell surface twice. They explained it, suggesting the existence of two layers of lipids at the cell boundary.

X-ray diffraction experiments of Schmitt and Palmer (Schmitt and Palmer, 
hydrophobic tails

hydrophilic head

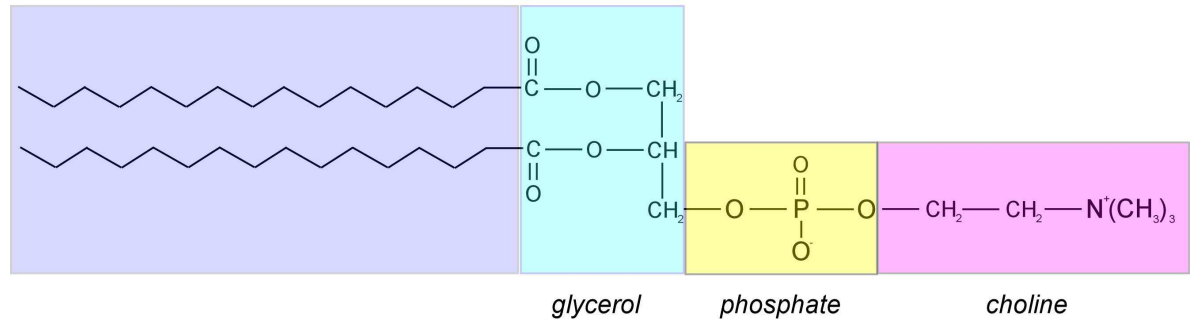

Figure 1.4: Chemical structure of a phospholipid 1,2Dipalmitoyl-sn-Glycero3-Phosphocholine, DPPC.

1940) from 1940-1941 confirmed this assumption. In the 1950-1960s electron microscopy (Robertson, 1957), (Branton, 1966) and X-ray diffraction experiments (Finean and Burge, 1963) gave strong evidence of the existence of the lipid bilayer at the cell boundary.

The "Fluid Mosaic" model, ( fig. 1.5 ), which was suggested by Singer and Nicolson (Singer and Nicolson, 1972), and its variants all include lipid bilayer as structural core of the membrane. In these models the lipid bilayer is a kind of quasi two- dimensional fluid matrix, in which swim proteins.

Later in the 1968-1978 the existence of a solid ordered state of the lipid membrane was shown by X-ray (Luzzati, 1968) and calorimetry studies. The solid ordered state characterized by ordered chains of individual lipids and presence of spatial order, when lipids form crystalline lattice. The fluid disordered state is characterized by disordered chains of individual lipids and the absence of a crystalline order.

From 1968 till now phase transitions were studied for different lipid systems. Among factors, which influence the phase transition of lipid membrane one can mention the length of the hydrocarbon chains. The longer the chains, the 


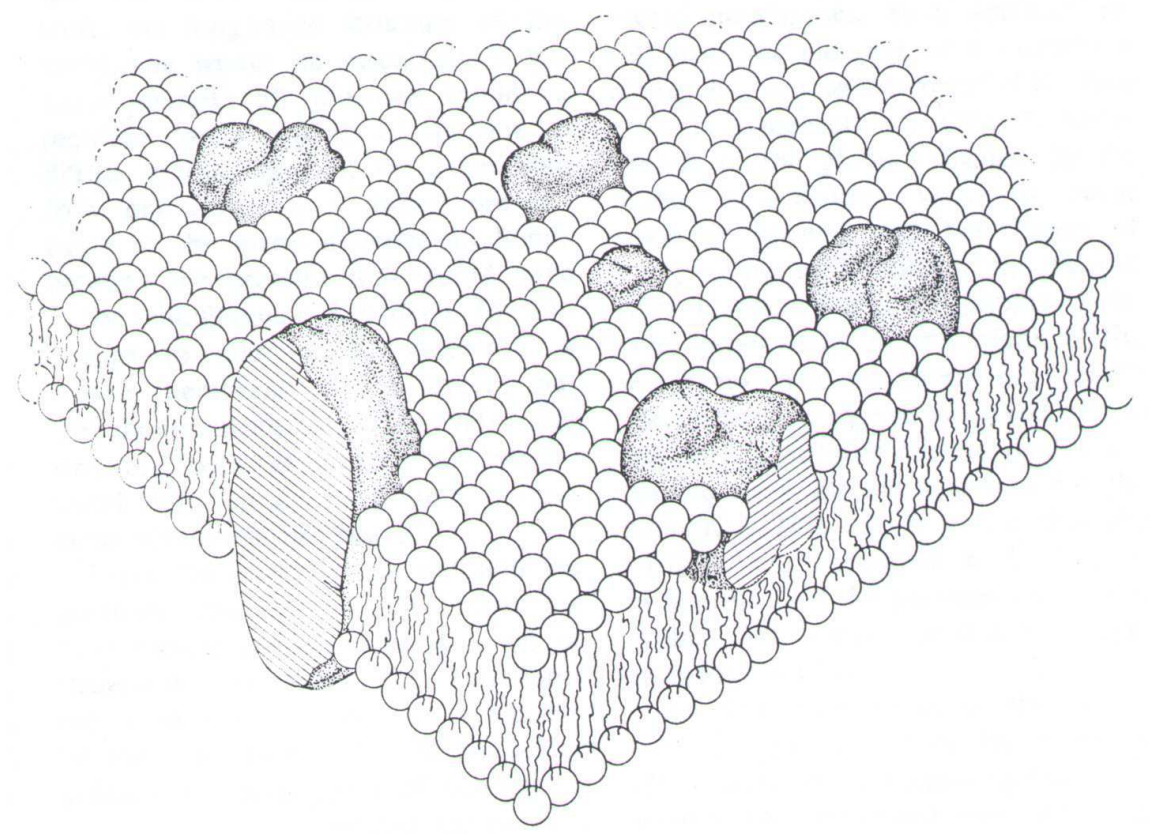

Figure 1.5: "Fluid Mosaic" model, suggested by Singer and Nicolson (Singer and Nicolson, 1972).

higher is the transition temperature (Landbrooke and Chapman, 1969). The presence of double bond decrease the transition temperature (Calhoun and Shipley, 1979). The type of head group influences the phase transition (Landbrooke and Chapman, 1969), as well as peptides (Ivanova et al., 2003) and cholesterol (Landbrooke et al., 1968).

Nonlamellar phases were studied as well, mostly by X-ray diffraction.

In 1984 Bloom and Mouritsen proposed so called "mattress model", ( fig. 1.6 ) which illustrates the "hydrophobic mismatch" principle of lipid-peptide interactions. Integral proteins have hydrophilic and hydrophobic parts. If the hydrophobic part is longer or shorter then the lipid bilayer it can influence the state of the lipids near the protein, because it is energetically unfavorable to expose hydrophobic parts to water. The lipid membrane becomes thinner 


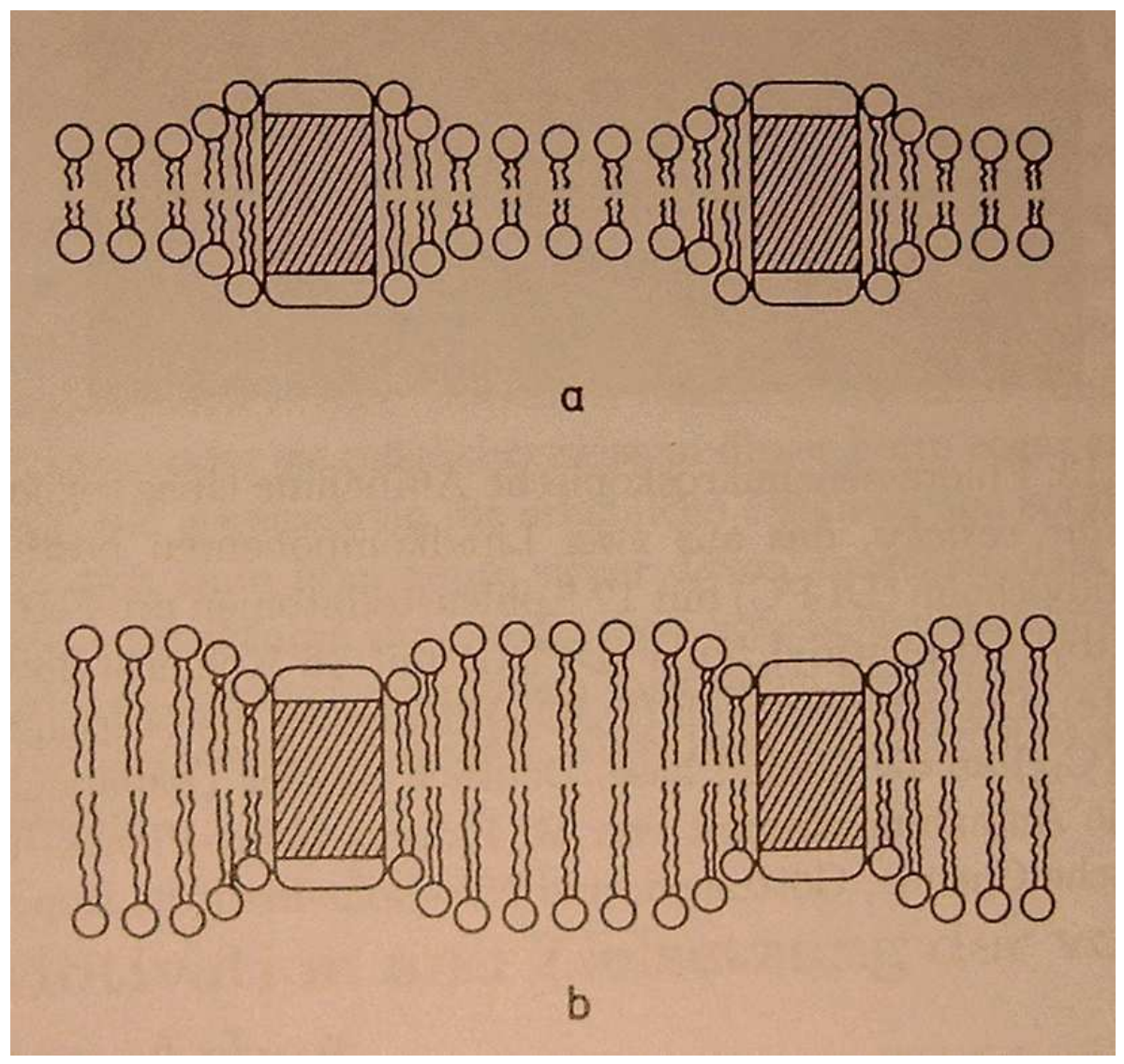

Figure 1.6: "Matress model", suggested by Bloom and Mouritsen. In (a) the protein is longer as the lipid bilayer, in (b) - shorter. Hydrophilic parts are white, hydrophobic - dashed. Lipids near protein take an energetically favorable state. (Picture from (Mouritsen and Bloom, 1984)).

or thicker near the peptide.

Mechanical properties of lipid bilayer, like bending rigidity, surface shear rigidity, area expansion moduli (Needham and Zhelev, 1996), thermal area expansivity, elastic area compressibility (Kwok and Evans, 1981) (Evans and Needham, 1987) was studied by micropipette aspiration experiments, as well by densitometry and other methods (Heimburg, 1998), (Ebel et al., 2001).

In 2000 AFM images of domains in the lipid membrane were obtained (Nielsen et al., 


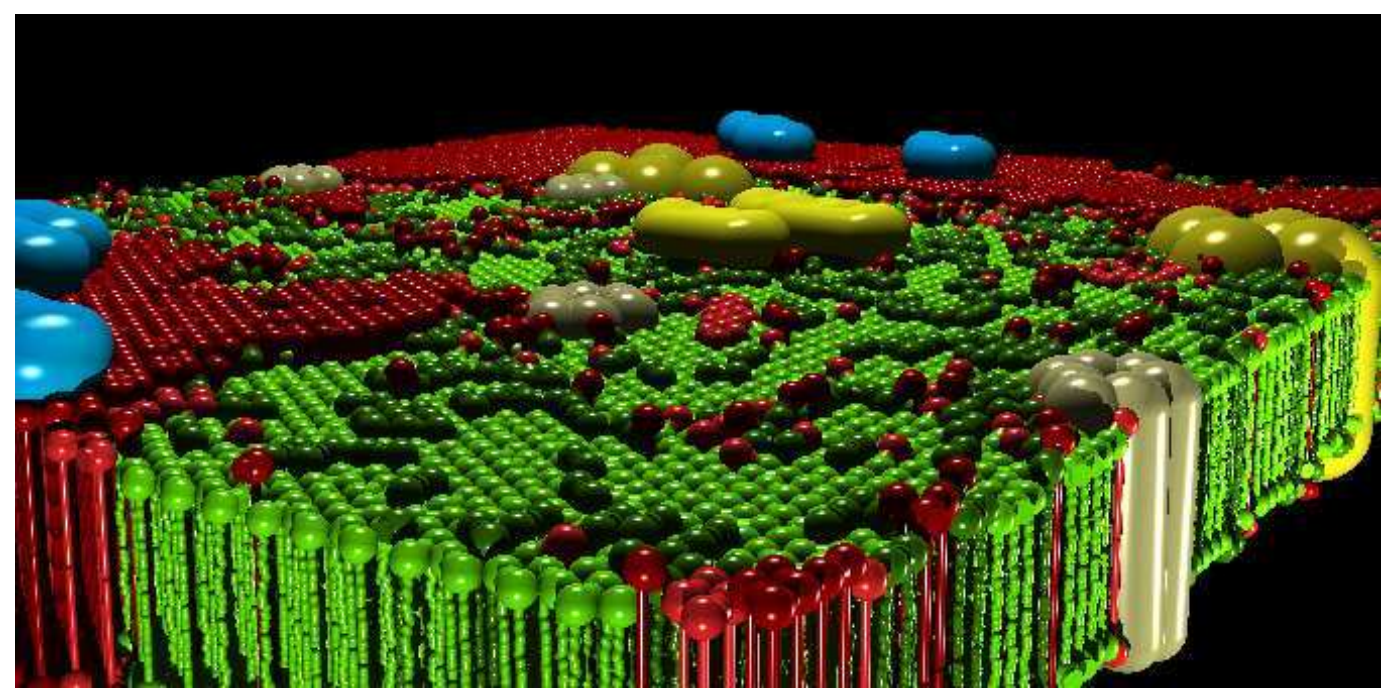

Figure 1.7: Modern picture of a biological membrane. The green color corresponds to liquid-disordered state, and red - to solid-ordered. Also transmembrane and peripheral proteins are shown. (Picture by H. Seeger).

2000).

Fig. 1.7 shows modern picture of a biological membrane. Here you can see different domains. The green color corresponds to liquid-disordered state, and the red refers to solid-ordered. Transmembrane and peripheral proteins are also shown.

\subsection{Membrane permeability : general view}

The lipid membrane is permeable for small hydrophobic molecules like $\mathrm{O}_{2}, \mathrm{CO}_{2}, \mathrm{~N}_{2}$, benzene, small uncharged polar molecules like $\mathrm{H}_{2} \mathrm{O}$, glycerol, ethanol, but is not permeable for lager uncharged polar molecules like amino acids, glucose, nucleotides, and ions like $\mathrm{H}^{+}, \mathrm{Na}^{+}, \mathrm{HCO}_{3}^{-}, \mathrm{K}^{+}, \mathrm{Ca}^{2}+, \mathrm{Cl}^{-}, \mathrm{Mg}^{2}+$. ( fig. 1.8 ) 


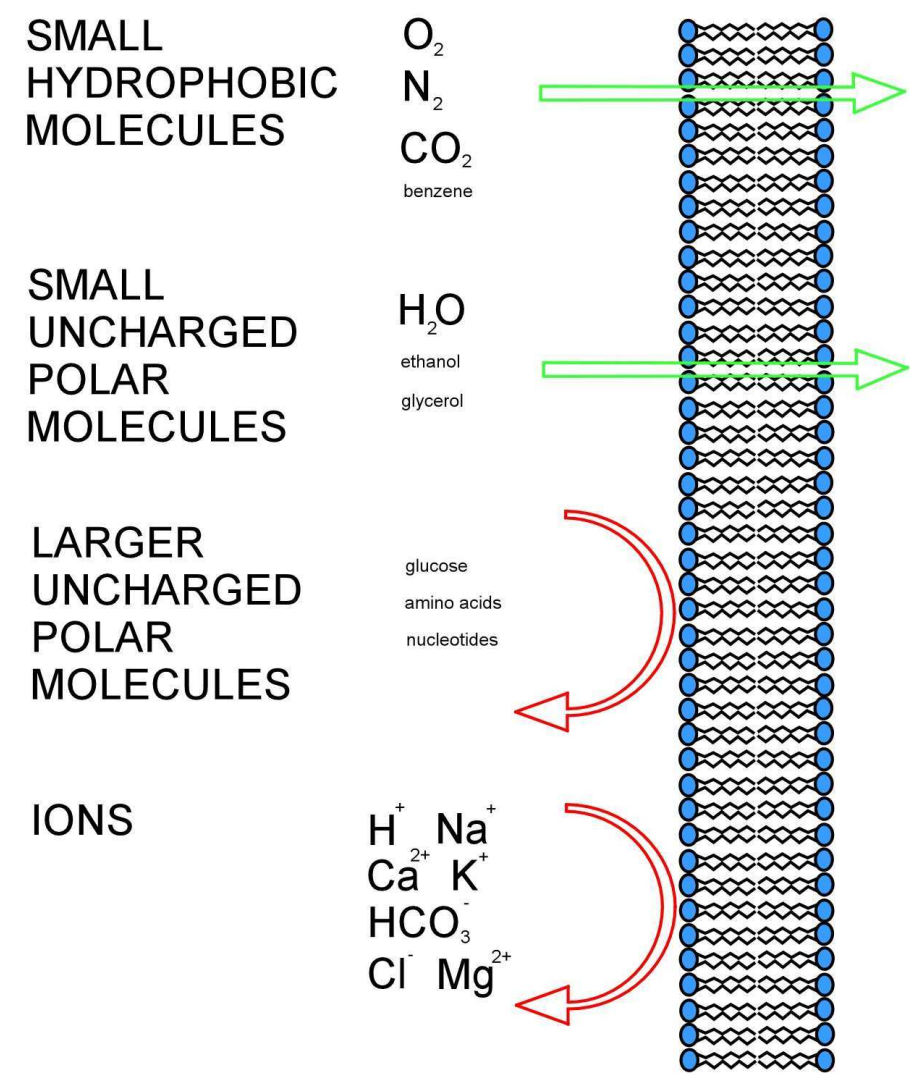

Figure 1.8: Permeability of a lipid membrane to small molecules.

There are different ways for a molecule to pass through the lipid membrane ( fig. 1.9 ). Permeation is a passive transport mechanism, when particles pass through the bilayer in direction of a concentration gradient, and to active transport, when particles go through the bilayer in direction opposite to the concentration gradient with the consumption of energy. The particle can simply diffuse through the bilayer, or use channel in proteins, or conformational changes in a carrier-protein.

If we put aside protein-mediated permeation, two basic models for perme- 


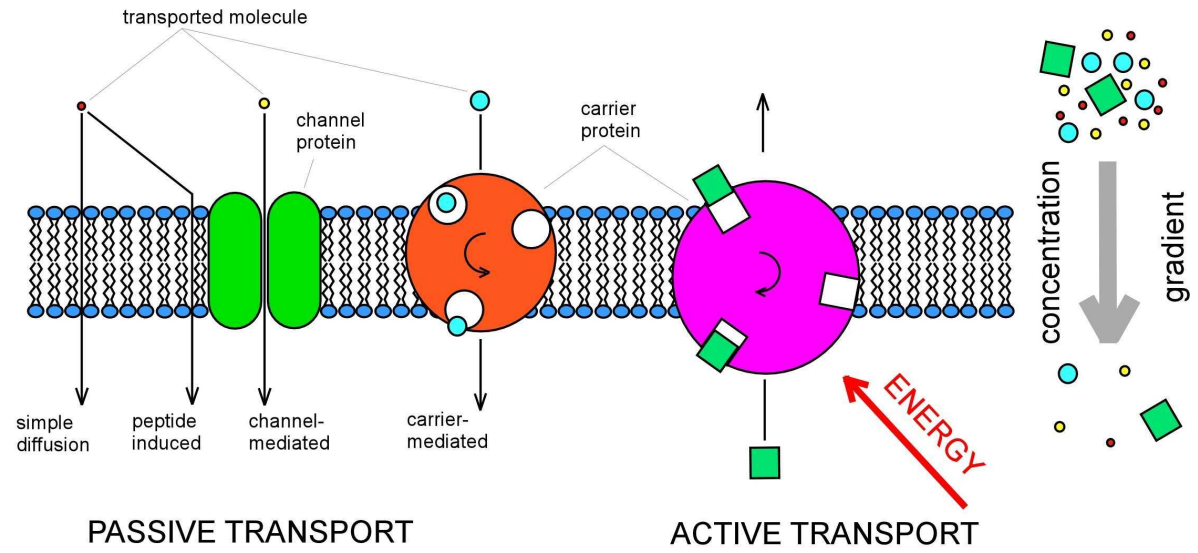

Figure 1.9: Passive and active transport - different ways for molecule to pass through the membrane.

ation of small particles can be considered : solubility-diffusion mechanism and permeation through the transient pores (Paula and Deamer, 1999).

In the solubility-diffusion model the hydrophobic region of the lipid bilayer is simplified to be a static area with sharp edges, which has properties of oil-like liquid, like octanol or ling-chain fluid hydrocarbon ( fig. 1.10 ). The permeating molecule first comes to this hydrophobic region, diffuses across it, and then leaves the membrane into the aqueous solution on the other side of the membrane. The change from the hydrophobic to the hydrophilic environment occurs abruptly. Using Fick's law of diffusion and the relationship between concentration differences inside and outside the bilayer permeability coefficients can be calculated. In this model it is not easy to decide, where to draw the boundary between aqueous and hydrophobic phases. This theory intends to predict permeation coefficients from known diffusion coefficients, partition coefficients and bilayer thickness.

This model describes well the diffusion of small neutral molecules, and can be applied to monovalent cations and halide ions, but must take into account 


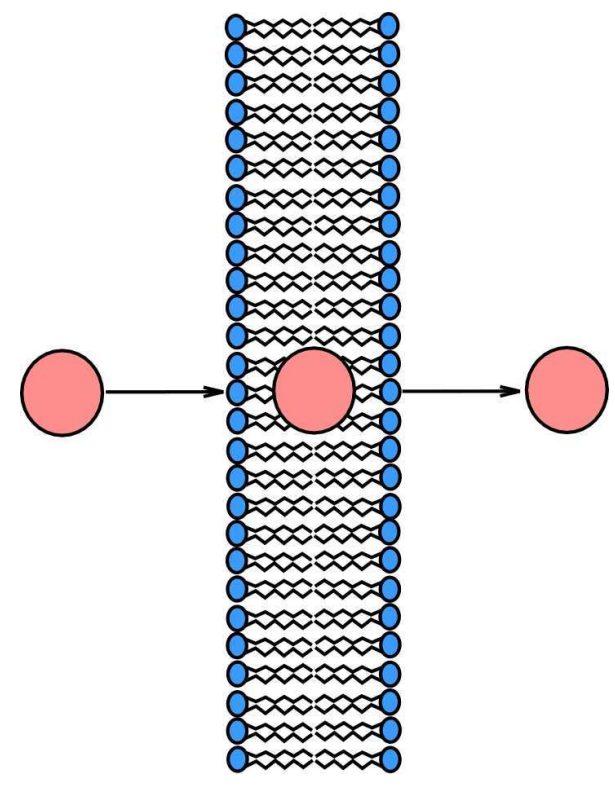

Figure 1.10: Solubility-diffusion mechanism..

other energy barriers. This model fails to describe cation transport through

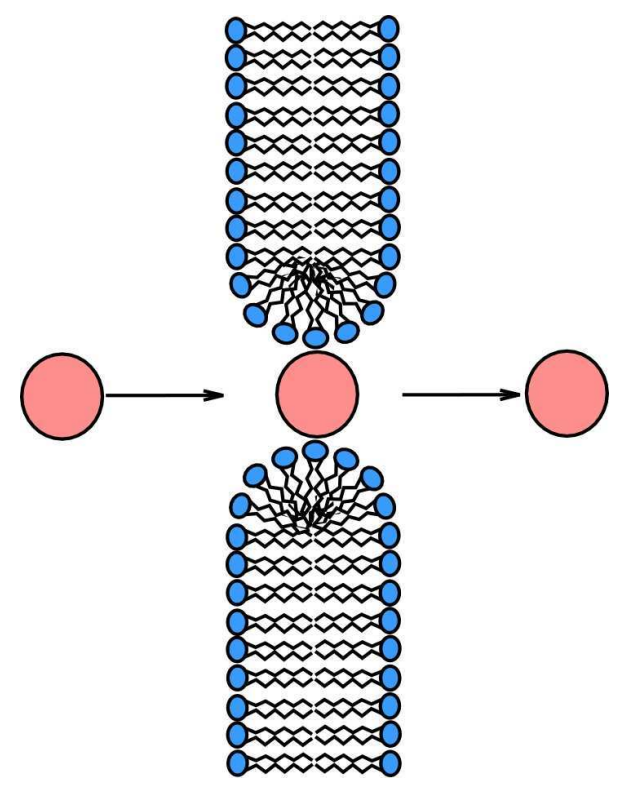

Figure 1.11: Transient pore permeation mechanism..

thin lipid bilayers, like potassium in phosphatidylcholines vesicles, so we need 
another mechanism : permeation through transient pores ( fig. 1.11).

Parsegian (Parsegian, 1969) demonstrated, that the energy of ion, located in transient pore is smaller as the energy of the same ion, located in the hydrophobic part of the lipid bilayer. Therefore, the diffusion barrier is lower and the permeation rate is higher. Pores are assumed to be hydrophilic rather than hydrophobic, because it takes less energy to create a hydrophilic pore as hydrophobic one. The permeation rate is related to the energy, necessary to create a pore of sufficient size and depth in bilayer (Hamilton and Kaler, 1990).

This mechanism can also be used to describe proton permeation through the bilayer.

In protein-mediated permeation two types of proteins are involved : channel proteins and carrier proteins (or pumps). Channel proteins facilitate diffusion in direction of concentration gradient and are part of the passive transport, as it was defined before. Pumps transport particles against concentration gradient using an external source of energy like ATP or light ( active transport ).

P-type ATPases and the ATP-binding cassette pumps use conformational changes on ATP binding and hydrolysis to transport bounded ion across the membrane. In $\mathrm{Ca}^{2}+$-ATPase and the $\mathrm{Na}^{+}-\mathrm{K}^{+}$-ATPase phosphorylation and dephosphorylation cause orientational and affinity changes of their ion binding sites.

The sodium-calcium exchanger is an example of different active transport mechanism. It uses the gradient of one ion to transport actively another one. In part one and three of this overview I followed ideas of Kleinzeller, expressed 
in his wonderful study of early permeability concepts (Kleinzeller, 1999), and McIntosh views on history of membrane structure understanding (McIntosh, 1999).

\subsection{Permeability models and simulation ap- proaches}

In 1978 Doniach (Doniach, 1978) introduced a two state Ising-like model of a lipid membrane and applied the Landau-Ginsburg approximation to calculate the membrane permeability. In his consideration he also suggested that the main phase transition of a lipid membrane could be of second order.

The same year Nagle (Nagle and Scott, 1978) considered the relation between lateral compressibility and bilayer permeability and used the OrnsteinZernike approximation for a two dimensional system. He predicted that membranes or parts of membranes may exist in near critical state in which large -scale fluctuations in lipid density could exist.

In 1988 Cruzeiro-Hansson and Mouritsen (Cruzeiro-Hansson and Mouritsen, 1988) used Monte Carlo simulation of the 10-state Pink model and predicted that permeability would be proportional to the length of domain interface.

The same year Shillcock and Seifert (Shillcock and Seifert, 1988) applied Monte Carlo simulations to a 2-D fluid of hard discs. They predicted existence of a critical density of small holes, where is transition from an equilibrium state containing many small holes to a state in which a large hole grows catastrophically.

In 2001 Groot and Rabone (Groot and Rabone, 2001) used a dissipative dynamics method to simulate the effect of surfactants on the lipid membrane. 
In this method several atoms were united into one simulation particle and effective interaction parameters are fitted to reproduce compressibility and solubility of various liquid components.

In 2003 Fournier and Joos (Founier and Joos, 2003) applied Monte Carlo simulations to a 2-D lattice model with multiple occupancy of the sites to simulate the kinetics of rupture of membranes under tension. The membrane was characterized by the bulk compressibility, the thickness of the hydrophobic part of the bilayer, hydrophobicity and rigidity of the tails. A phase diagram for rupture was presented.

In 2004 Leontiadu et al. (Leontiadu et al., 2004) presented Molecular Dynamics simulation of hydrophilic pore formation process in lipid bilayer. A critical threshold tension was estimated, as well the structure and size of pores were characterized.

\subsection{Phase transition, local fluctuations and permeability}

Let us consider the main phase transition of lipid membrane in some more detail.

Phospholipid ( DPPC ) membrane undergoes the main phase transition from gel ( solid - ordered ) to fluid ( liquid - disordered ) state at $41^{\circ} \mathrm{C}$. Below this temperature the membrane is in a gel ( solid - ordered ) state which is characterized by ordered chains of each individual lipid ( they lie in an energetically favorable all-trans conformation ( fig. 1.12 )) and on lipids organization in kind of two dimensional crystalline lattice. The word solid refers to the state of the single lipid and the word ordered describes the 


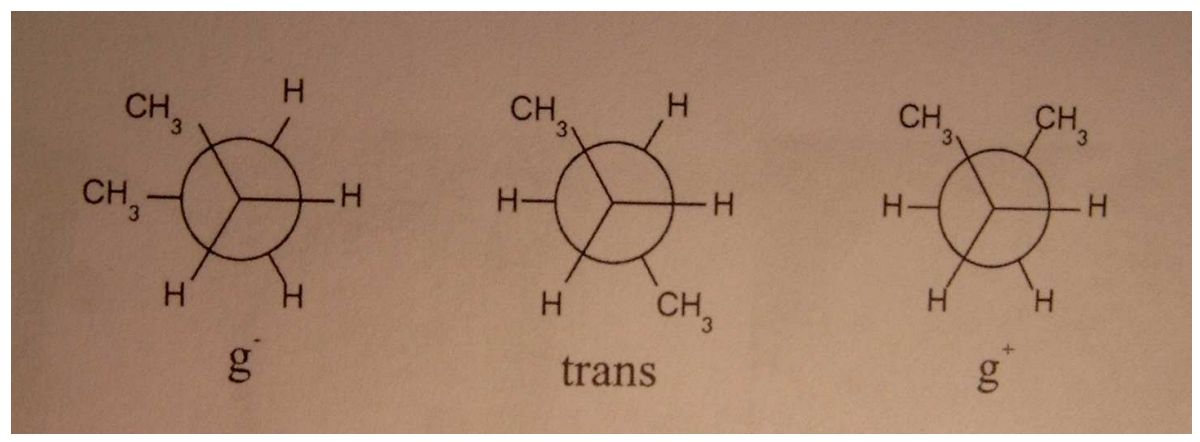

Figure 1.12: Illustration of trans and $g^{-} g^{+}$isomers of Ethan molecule. Exactly these conformations show segments of lipid chains ( $\mathrm{CH}_{2}$ groups are in the place of shown $\mathrm{CH}_{3}$ groups).

crystalline order of the lipid matrix. Above this temperature membrane is in a fluid ( liquid - disordered ) state. In this state lipid chains are flexible and have $g^{-}$and $g^{+}$conformations ( fig. 1.12 ) and there is no more crystalline order present. Fig. 1.13 shows the fluid $L_{\alpha}$ phase and different gel phases.

Examples of heat capacity profile for this kind of phase transition is shown in fig.1.14. The half - width of the transition of the pure lipid membrane is about $1^{\circ} \mathrm{C}$. If each lipid melted independently the half - width of such a transition would be about $60^{\circ} \mathrm{C}$ (Gennis, 1989). This means that lipid melting is a collective process, lipids melt not independently of each other but in a correlated manner. This is called cooperativity of the phase transition, and the transition half - width is the measure of the cooperativity. This phase transition is assumed to be of first order, however is actually close to a critical temperature, therefore thermal fluctuations are very strong. Large critical density fluctuations close to a phase transition point create heterogeneities and may result in lowering free energy barrier to particle diffusion (Doniach, 1978).

Therefore, the elastic constants of the membrane change dramatically near 


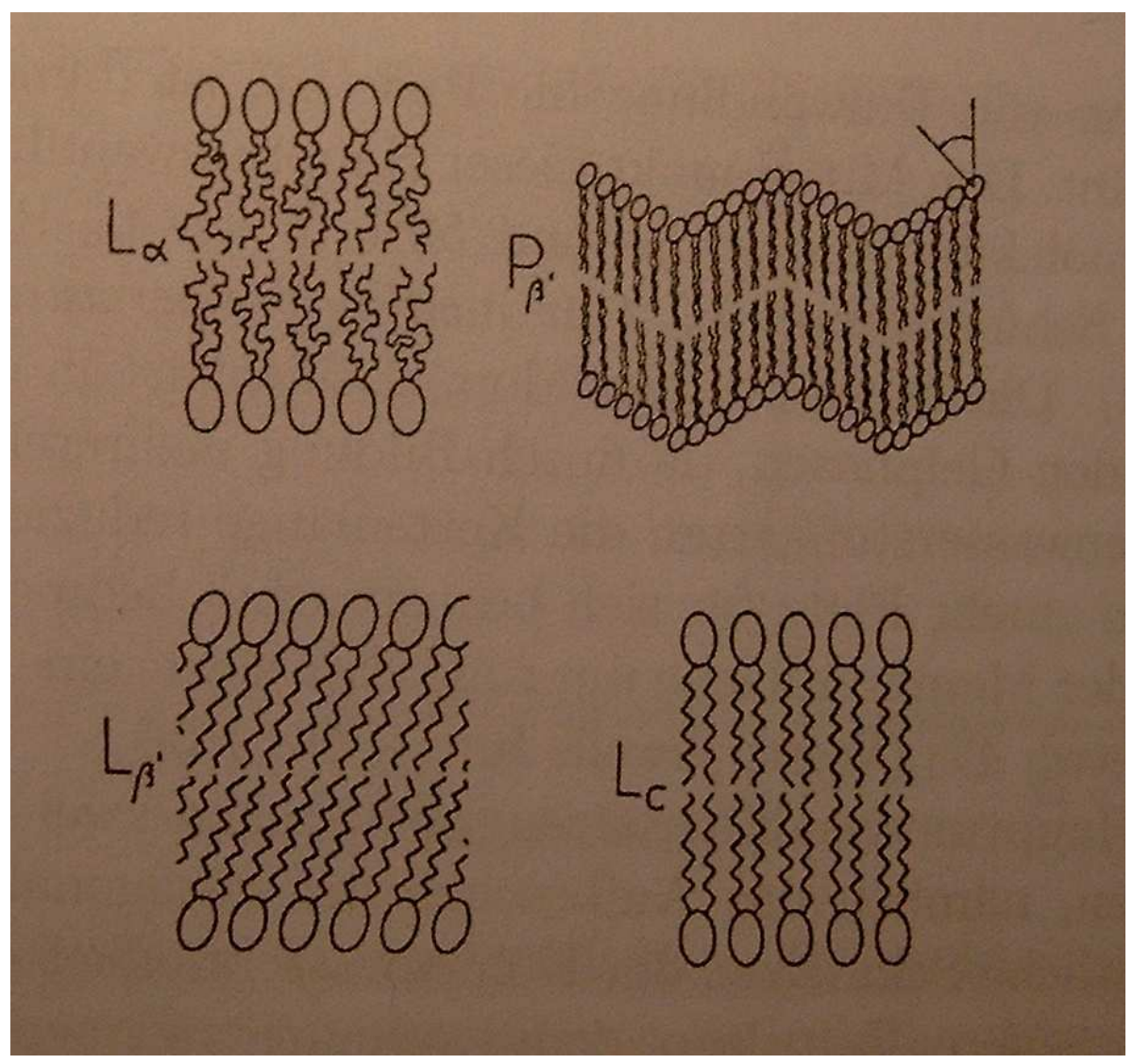

Figure 1.13: Fluid $L_{\alpha}$ phase and different gel phases. Picture by E. Sackmann, Technische Universität München..

the main phase transition (Heimburg, 1998). The membrane permeability increases about two orders of magnitude, measurement by (Papahadjopoulos et al., 1973). However, this measurement was done for a lipid membrane, which contains huge amount of cholesterol $30-60 \%$. Cholesterol decreases the cooperativity of transition and makes impossible study of cooperativity related effects, like caused by addition of peptides what is especially interesting for us.

Phase transitions are not only a feature of artificial lipid membranes, but occur as well in biological membranes of real cells. Some bacteria, like E.coli 


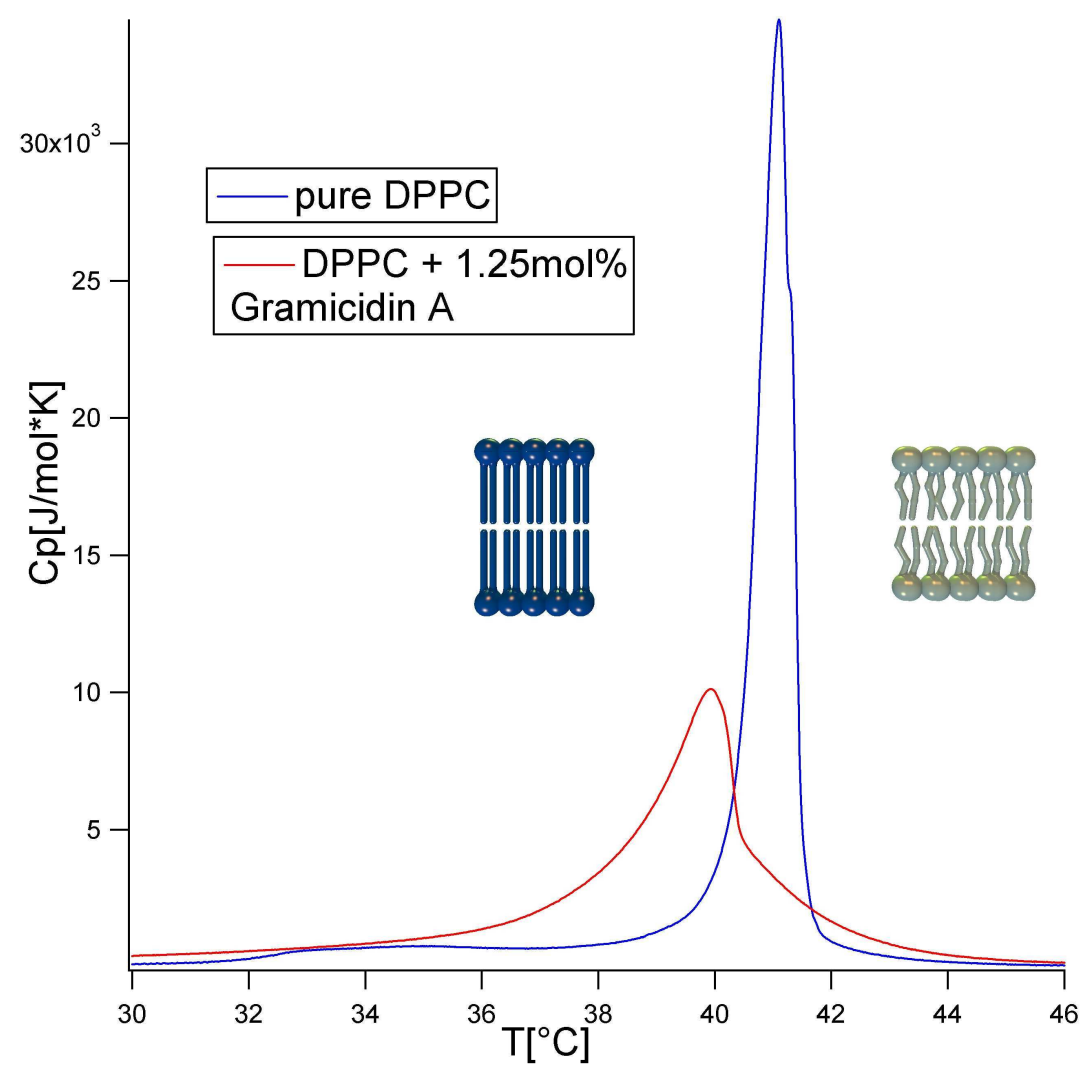

Figure 1.14: Heat capacity profile of a pure lipid membrane and of a peptide / lipid mixture. Addition of the peptide Gramicidin A shifts the phase transition temperature down and makes profile broadener.

show phase transition just below the temperature they grow at (Pollakowski, 2003).

The main phase transition can be influenced by many factors. Addition of peptides can shift the transition temperature up or down or broadens the profile ( fig. 1.14 ). Changes of pressure, $\mathrm{pH}$ also influence the phase transition. (Pollakowski, 2003)

Addition of peptides creates new heterogeneities and changes distribution of fluctuations. 
Fluctuations of local lipid environment are high near peptides and domain borders( fig. 1.15 ) (Ivanova et al., 2003).

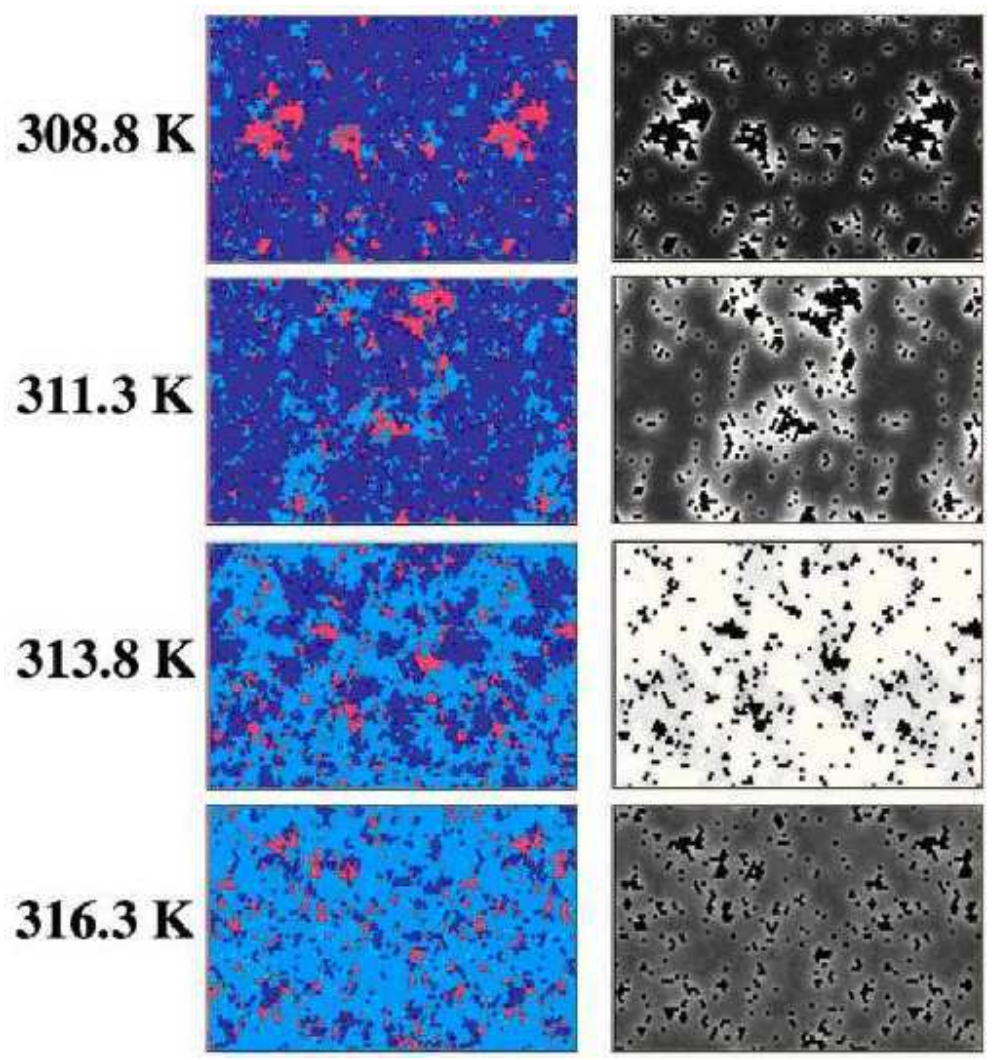

Figure 1.15: Local lipid fluctuations are high near peptides. They are represented on the left part of the image. White color corresponds to high fluctuations, black to small ones. On the right one can see solid-ordered (dark blue) and liquid-disordered (bright blue) domains. Peptides are represented by red color.

The fluctuations become extremely big when approaching the phase transition, and this, when considering this fluctuations as area fluctuations, causes dramatic increase of the membrane permeability.

When peptides influence the phase transition and the distribution of fluctuations, we expect them to influence the permeability as well. 
Study peptide influence on the membrane permeability experimentally and simulate this, using local lipid fluctuations based approach is the main aim of this work. 


\section{Chapter 2}

\section{Experimental Methods}

\subsection{Differential Scanning Calorimetry}

Differential scanning calorimetry (DSC) is used to study processes, which go along with consumption of energy, like phase transitions in membranes or protein denaturation. Data, obtained from DSC gives us information about the thermodynamics of these processes.

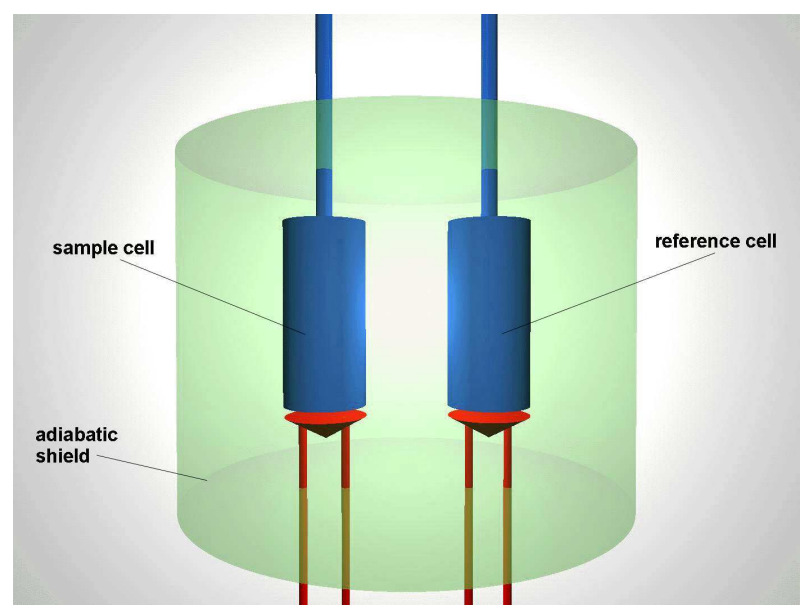

Figure 2.1: Schema of a Differential scanning calorimeter.

A differential scanning calorimeter ( the one which is used in our experiments 
is described in (Plotnikov et al., 1997) ) consists of two coupled together cells, which are isolated from the environment by adiabatic shell ( fig. 2.1 ). One of these cells is filled with a sample substance and the second with reference substance, usually the same solution, in which the sample in the sample cell is diluted. During a DSC scan both cells are heated simultaneously, keeping the temperature difference between the cells to be zero. The sample cell takes more heat because of studied thermodynamic process in the sample substance. This excess heat $\Delta Q$ is measured and recorded as a function of time $t$ and temperature $T$. With it's help one can calculate the heat capacity of the sample.

$$
\begin{aligned}
& \Delta Q=\int_{t}^{t+\Delta t} \Delta P\left(t^{\prime}\right) d t^{\prime} \cong \Delta P \cdot \Delta t \\
& \Delta C_{p}=\left(\frac{\partial Q}{\partial T}\right)_{p} \cong \frac{\Delta Q}{\Delta T}=\frac{\Delta P}{\left(\frac{\Delta T}{\Delta t}\right)}
\end{aligned}
$$

whereby $\left(\frac{\Delta T}{\Delta t}\right)$ is the scan rate and $\Delta P$ is the power difference between the sample and reference cells. The used VP-DSC calorimeter from MicroCal (NorthHampton/MA USA) has scan rates $0-60 \mathrm{deg} / \mathrm{h}$, a good signal to noise ratio and a stable base line. The coin-formed cells in this calorimeter are made of tantal, which has corrosion properties similar to glass. Peltier elements are used to heat or cool the cells. This calorimeter is a very sensitive and easy to handle tool.

\subsection{Probe preparation for Differential Scan- ning Calorimetry}

Doing calorimetric experiments $10 \mathrm{mM}$ lipid solutions were used. Lipids were purchased from Avanti Polar Lipids (Birmingham, AL) and Gramicidin A ( 
fig. 2.2 ) from Fluka (Buchs, Switzerland).

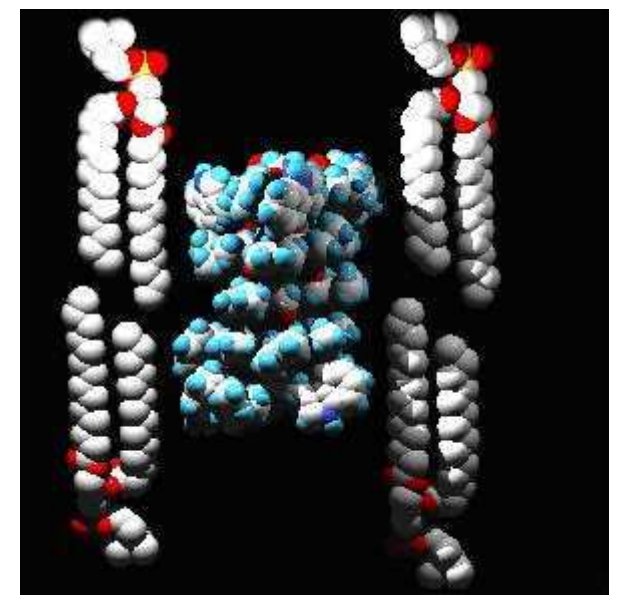

Figure 2.2: Gramicidin A peptide. (Picture by T.Heimburg, based on the NMR data from (Ketchem et al., 1993)).

Most of the experiments was done with unilamellar vesicles. They were obtained using extrusion of mulilemellar vesicles through a polycarbonate membrane with a pore diameter of 100nm in an extruder from Avestin (Ottawa,Canada).

A special device was designed to automatize the extrusion process ( fig. 2.3 ). It is possible to change the speed of the extrusion, or the time needed to push $1 \mathrm{ml}$ of solution through the polycarbonate membrane. This mechanical system puts always the same pressure on the filter membrane, and therefore helps in avoiding the breaking of the filter. A special counter is introduced to count the number of runs.

Lipid-peptide mixtures were first dissolved in organic solvent, then after evaporation of the solvent it was put into a dessicator for 10-12 hours.

Measurements were done in a $7.5 \mathrm{pH} 10 \mathrm{mM}$ Hepes 1mM EDTA buffer. 


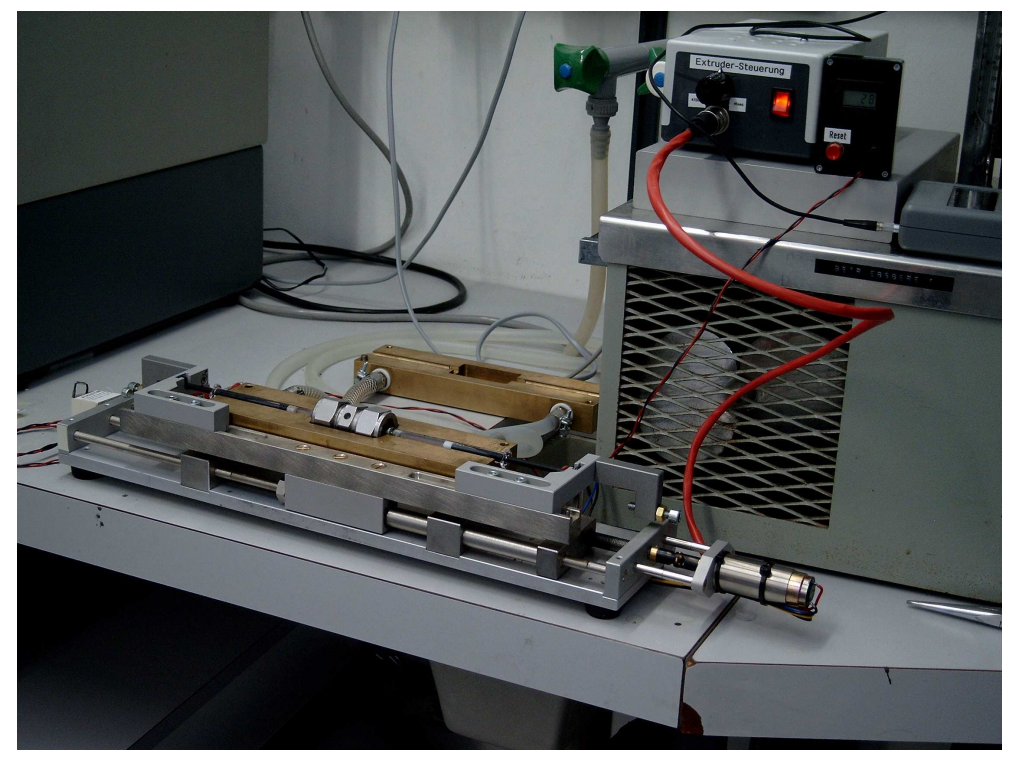

Figure 2.3: Special device, designed to automatize the extrusion process. It is possible to change the speed of the extrusion, or the time, needed to push 1 $m l$ of solution through the polycarbonate membrane. This mechanical system puts always the same pressure on the filter membrane, and therefore helps in avoiding the breaking of the filter. A special counter is introduced to count the number of runs.

\subsection{Fluorescence Correlation spectroscopy}

Fluorescence Correlation Spectroscopy (FCS) is a suitable tool to investigate permeation problems because of its high sensitivity, the small detection volume, and the molecular specificity - possibility to distinguish molecules of interest among a large number of different molecules (Rigler and Elson, 2001).

Our fluorescence correlation spectroscopy setup ( fig. 2.4) consists of a laser, an objective, a beamsplitter, a pinhole, two avalanche photodiodes, lenses and filters.

Laser light travels from the laser ( Nd:Yag $532 \mathrm{~nm}$ ) through two lenses ( $\mathrm{f}$ 


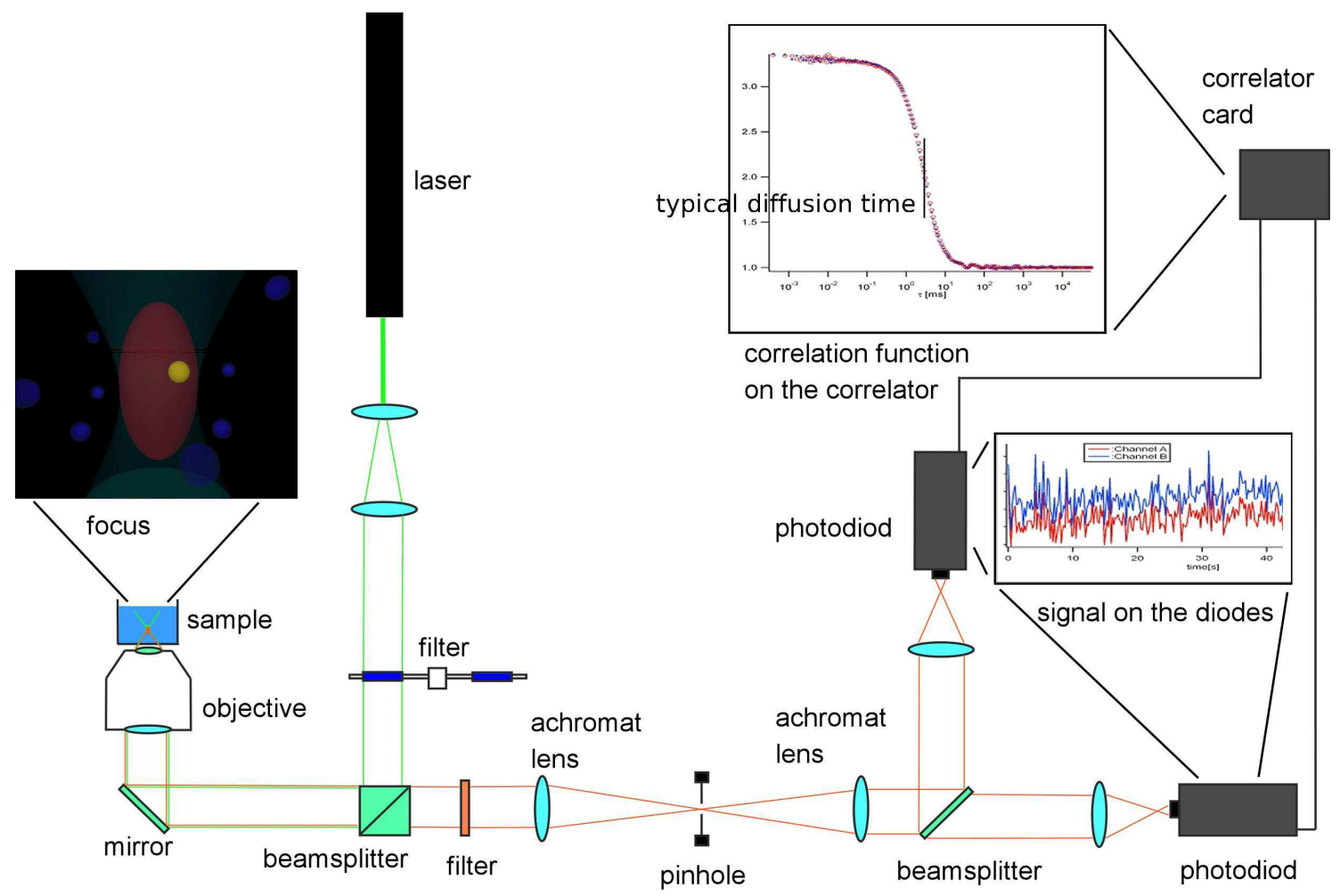

Figure 2.4: Fluorescence Correlation Spectroscopy setup.

$=5 \mathrm{~mm}$ and $\mathrm{f}=100 \mathrm{~mm}$ ) which broaden the laser beam and intensity filter to the objective (Olympus, UPLAPO $60 \times \mathrm{W}$, N.A. : $1.20, \mathrm{f}=3 \mathrm{~mm}$ ) . The sample is located in the focus of the objective. The laser light excites the dye molecules in the focus and they emit fluorescence light, which travels through the beamsplitter ( $537 \mathrm{~nm}$ ), which is transparent to the fluorescence light in direction from objective to diodes, but not to the laser light. The fluorescence light which propagates from the objective to the diodes goes through the filter ( T: $542-622$ ), which cuts off the rest of the laser light and the Rahman scattering from the water, and the pinhole, which defines the confocal volume. The diodes monitor the fluorescence intensity. The signal from the diodes is correlated in real time by a Flex 5000 corellator card ( correlator.com ), 
which is connected to a computer.

An automatization of the FCS measurement process was realised, through the usage of a script language. This script allows to control a shutter, which is between laser and first lens and it controls the correlator card, as well. This allows measurements with fixed time intervals in between the measurements and combined with a water supply system, which is designed to prevent the dry out of a drop of water between the objective and the probe allows the extension of the experiment time to several days.

A correlation function can be introduced following Eigen (Eigen and R., 1994), using the intensity $I(T)$ as monitored from the diode which fluctuates around a mean value $\langle I\rangle$ with the deviation $\delta I$ :

$$
G(t)=\lim \frac{I}{2 T} \int_{-T}^{T} I\left(t+t_{1}\right) I(t) d t_{1}=\langle I(t) I(0)\rangle
$$

$G(t)$ can be divided into two parts: a constant term $\langle I\rangle^{2}$ and a time dependent term $\langle\delta I(t) \delta I(0)\rangle$. Considering the Poisson-distributed statistics of small numbers and normalizing $G(t)$ by $\langle I\rangle^{2}$ it shows that the limit of the amplitude of the time dependent term at $G(t=0)$ is given by the inverse number of molecules $\mathrm{N}$ within the volume element :

$$
\lim \left[\langle\delta I(t) \delta I(0)\rangle /\langle I\rangle^{2}\right]=1 / N
$$

The autocorrelation was invented by Norbert Wiener as a powerful mathematical tool for noise reduction.

In our case correlation function gives us the typical diffusion time of the diffusing particle ( fig. 2.4 ), it gives us information about how fast or how slow particles moves in the focus and this is exactly what we need for our membrane permeability measurements. 
a)
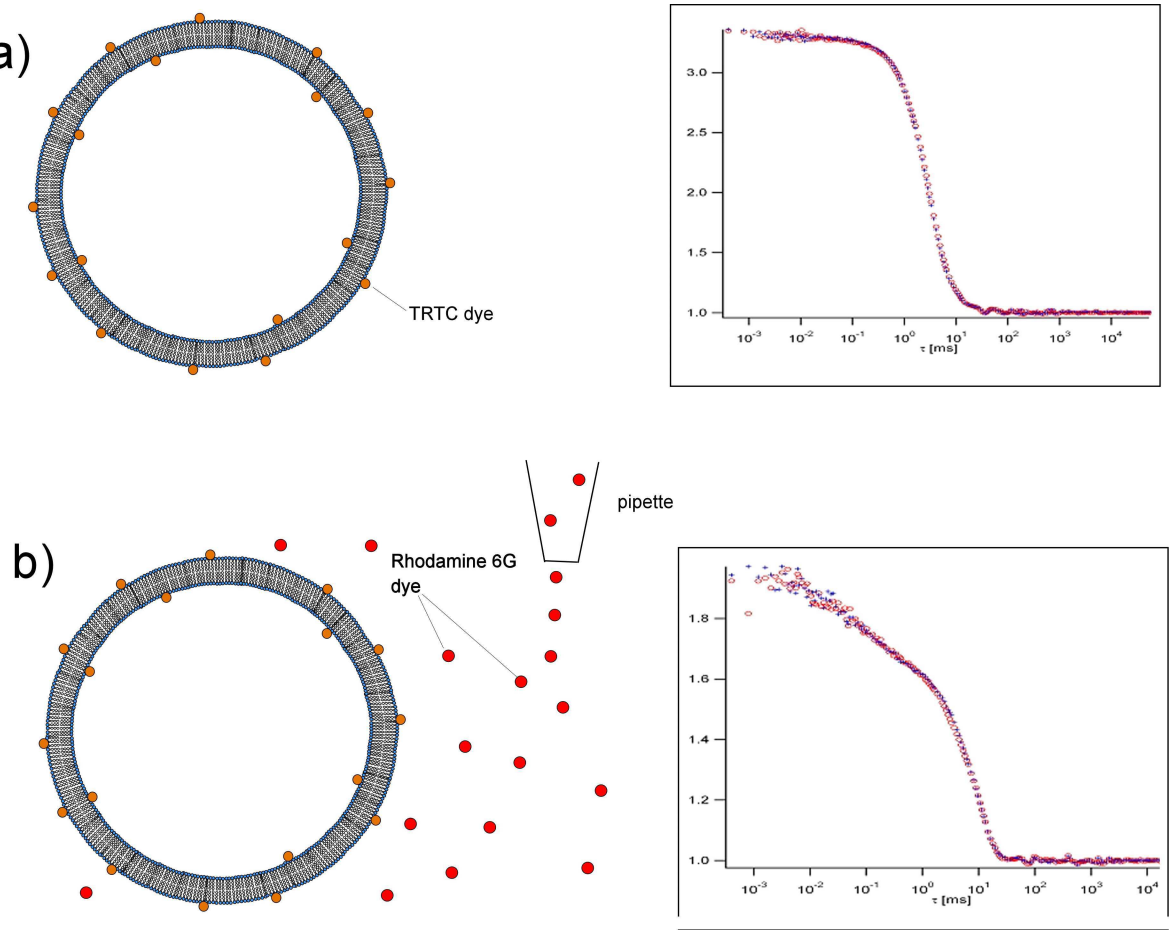

Figure 2.5: First test experiment. A solution, containing unilamellar lipid vesicles with integrated TRITC dye is put into the confocal volume of the FCS setup, and a FCS curve, which is typical for vesicles is measured (a). Then keeping this vesicles solution free particles of Rhodamine $6 G$ dye are added. Adding small portions of the solution, which contains Rhodamine 6 G particles results in a changes in FCS profile (b).

Several test and control experiments were done with the aim to establish the measurement technique.

At first unilamellar lipid vesicles with integrated TRITC dye were prepared. TRITC is a lipid with a dye sequence attached to the headgroup. It integrates well into the membrane, but does not swim freely in a water solution, like it does Rhodamine $6 \mathrm{G}$ ( fig. 2.5 ). The chemical structures, formulas and molecular weights of the used dyes can be found in attachment B.

Then solution, of TRITC integrated unilamellar vesicles is put into the con- 
focal volume of the FCS setup. A FCS curve of these vesicles is measured and the typical diffusion time is obtained.

Then keeping this vesicles solution in confocal volume of FCS setup free particles of Rhodamine $6 \mathrm{G}$ dye are added. Rhodamine $6 \mathrm{G}$ is a small molecule and diffuses freely in a water solution. Therefore Rhodamine $6 \mathrm{G}$ has shorter diffusion time in comparison to the vesicles. Adding a small portions of the solution, which contains Rhodamine $6 \mathrm{G}$ particles, changes of the FCS profile and its intensity are observed. The intensity change corresponds to the change of the number of the fluorescent dye particles in the confocal volume, assuming no bleaching and dye absorption on the walls of the measurement cell is taken into account. Another assumption is that two vesicles, containing an amount of dye $A$ and $B$ give the same contribution to the FCS profile as one vesicle with dye amount $A+B$, and the dye used for vesicles and free labels have the same quantum yield.

In another experiment when small portions of solution, containing TRITC -labeled vesicles were added to a Rhodamine 6G solution.

A two-component fit to the FCS profiles is used to obtain relative populations of vesicles and free particles.

The two component correlation function formula for 3D from (Rigler and Elson, 2001) is used :

$$
\begin{aligned}
G(\tau)=1+\frac{1}{N}[ & (1-y)\left(\frac{1}{1+\frac{\tau}{\tau_{D 1}}}\right)\left(\frac{1}{1+\left(\frac{\omega}{z}\right)^{2} \frac{\tau}{\tau_{D 1}}}\right)^{\frac{1}{2}} \\
& \left.+(y)\left(\frac{1}{1+\frac{\tau}{\tau_{D 2}}}\right)\left(\frac{1}{1+\left(\frac{\omega}{z}\right)^{2} \frac{\tau}{\tau_{D 2}}}\right)^{\frac{1}{2}}\right]
\end{aligned}
$$

where $N$-number of particles in the focus, $\omega, z$ - focus parameters, $\tau_{D 1}, \tau_{D 2}$ 
- diffusion times for particles of sort 1 and 2 respectively, $(1-y)$-fraction of particles of sort 1, $y$-fraction of particles of sort 2 .

Then, I plot the intensity ratio as a function of fraction of the slow component, obtained from the fit of the FCS profiles ( fig. 2.6 ).

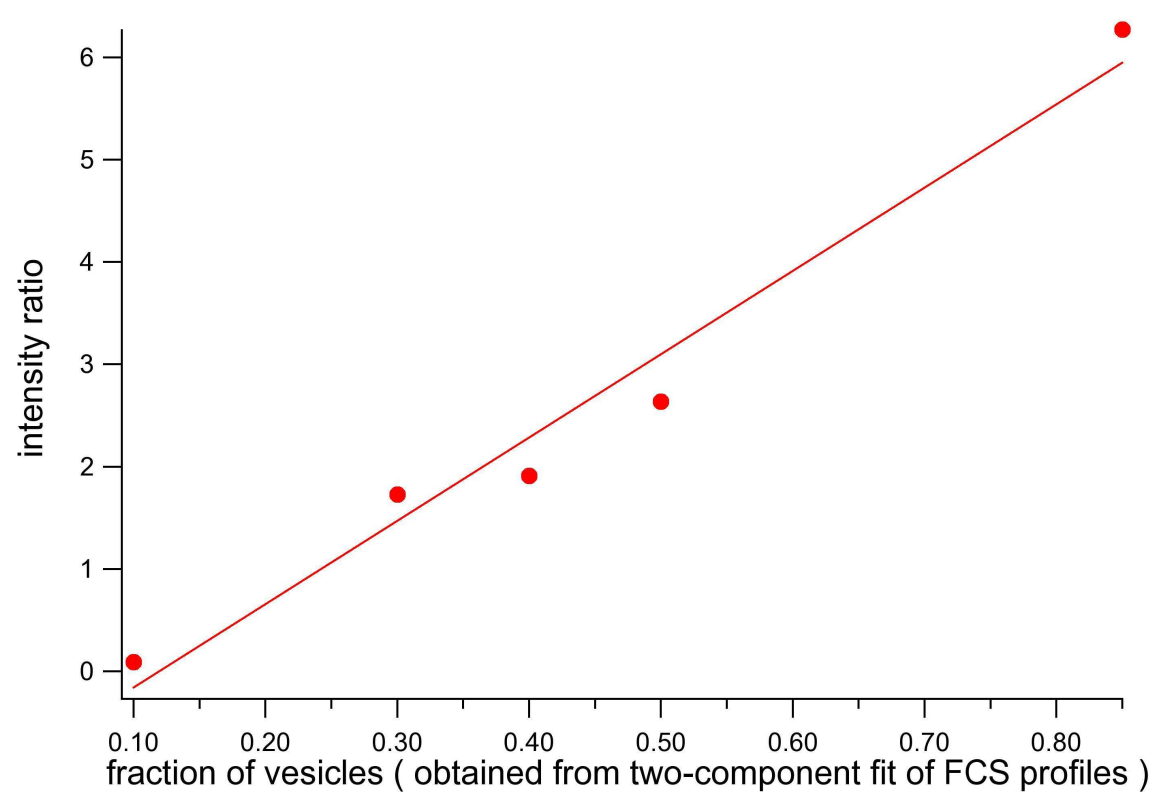

Figure 2.6: Intensity ratio as a function of the fraction of the slow component, obtained from the fit of the FCS profiles. The obtained linear dependence justifies the use of a two-component fit formula for the estimation of the relative populations of the slow and fast components, assuming no bleaching and dye absorption on the walls of the measurement cell.

The obtained linear dependence justifies the use of a two-component fit formula for the estimation of the relative populations of the slow and fast components, assuming no bleaching and dye absorption on the walls of the measurement cell.

From this experiment we know, that we can distinguish between the vesicles and the free particles and how to obtain relative populations of vesicles and free particles from fitting FCS curves. 
a)
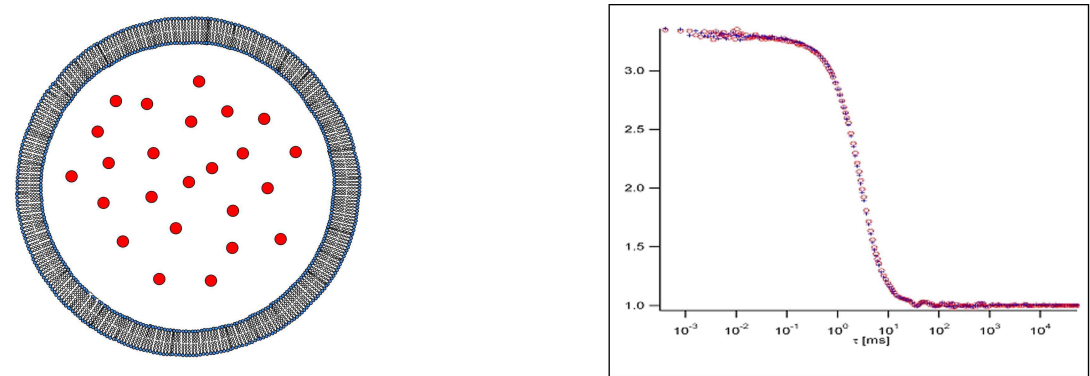

b)
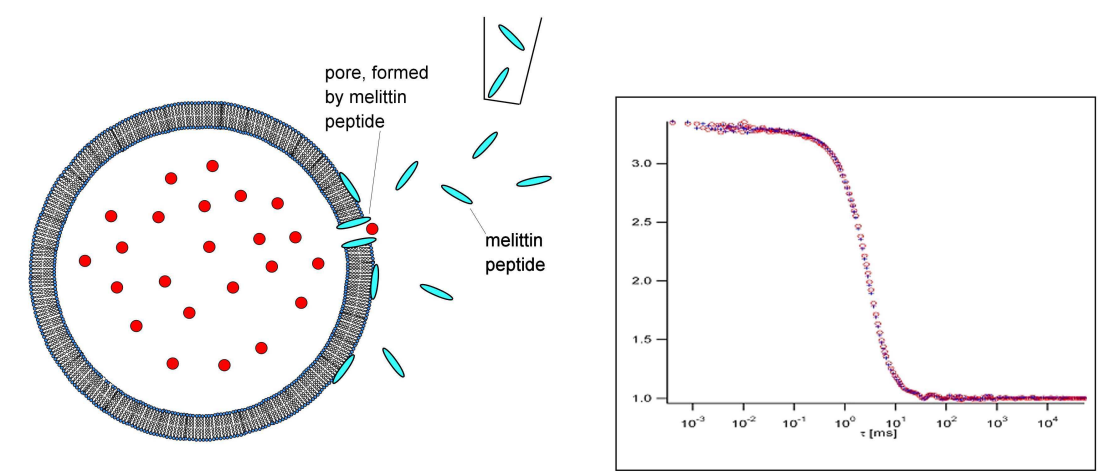

c)
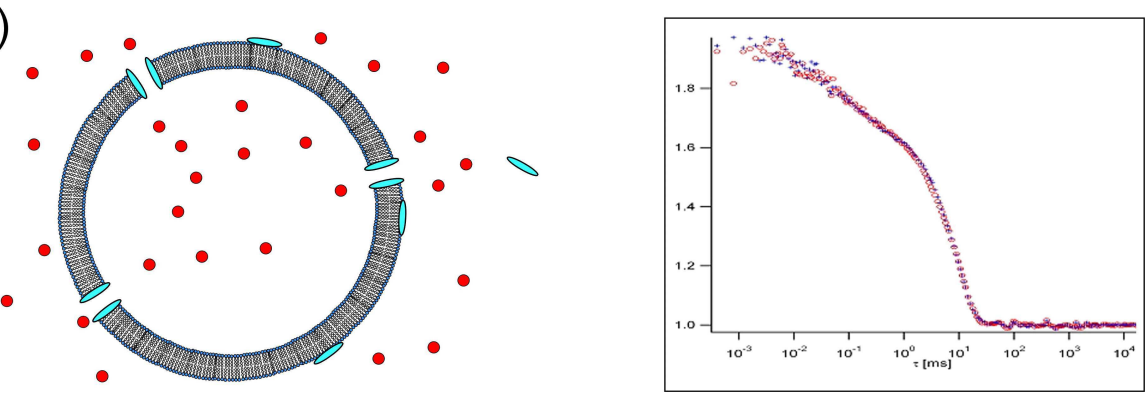

Figure 2.7: Illustration of permeation, caused by a pore-forming peptide,like melittin. Solution, containing the vesicles with dye inside was put into the focus of the FCS setup. I observed curves typical for vesicles. (a) Then a small amount melittin solution was added. Melittin peptides incorporate into the membrane and build pores. (b) Through these pores Rhodamine dye particles come to the outside and I observe the appearance of a fast diffusing component (c).

An other experiment was done with the aim to prove that free Rhodamine particles do not stick to the lipid vesicles. 
Unilamellar vesicles with no dye were prepared. Then they were put into the focus of the FCS setup. Then we added free Rhodamine dye particles, and observed only FCS profile, which are typical for free particles.

Another experiment consists of observing permeation, caused by pore-forming peptides, like melittin ( fig. 2.7 ). Unilamellar vesicles with Rhodamine 6G dye inside were prepared using extrusion. The dye outside the vesicles was washed out using chromatography. The solution, containing the vesicles with dye inside was put into the focus of the FCS setup. I observed curve typical for vesicles.

Then a small amount of melittin solution was added. Melittin peptides incorporate into the membrane and form pores. Through these pores Rhodamine dye particles come to the outside and I observe the appearance of the fast diffusing component. Such measurements were done for different concentrations of melittin. In this experiment the measurement technique was established, and different permeation kinetics were observed for different melittin concentrations. fig. 2.8 shows kinetics of permeation induced by addition of melittin-like peptide.

This experiment was done only with the aim to establish experimental method, therefore exact concentrations of the melittin were not determined.

A series of control experiments were done to prove the stability of the vesicles. Usually unilamellar vesicles are not stable, they fuse, then breake down therefore releasing the dye. To avoid this a small amount of charged lipid was added ( fig. 2.10). This prevents diffusion and makes the vesicles stable. A solution, containing vesicles with the dye inside was kept at room temperature for several days and periodically controlled in a FCS experiment. The vesicles were stable and no dye leakage was observed. 


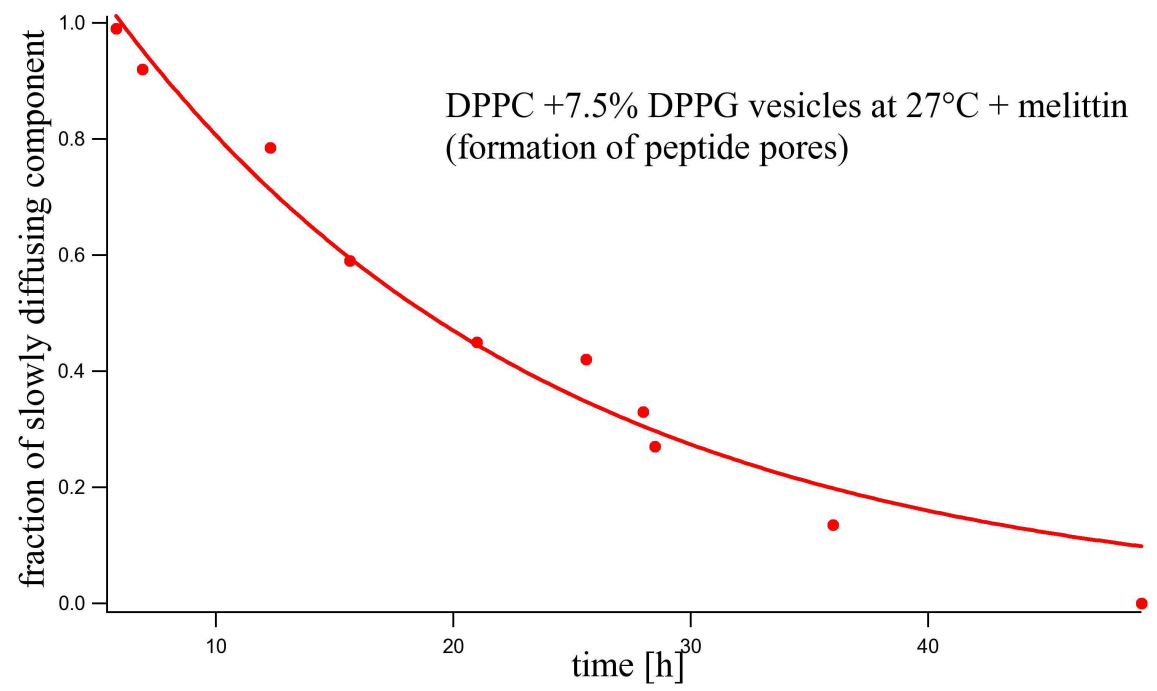

Figure 2.8: Kinetics of permeation induced by addition of melittin-like peptides.

Such a stability plays a crucial role when measuring permeability at high temperatures. Achieving such a stability makes it possible to measure permeability dependence on temperature.

The probe preparation is described in detail in the next section.

In my permeation experiment ( fig. 2.9) we have two sorts of particles big and slow vesicles and small and fast free dye particles. ( fig. 2.9 a) ) shows a correlation function typical for a solution where mostly vesicles diffuse and ( fig. 2.9 c) ) for a solution with mostly free particles.

First I put dye particles inside the vesicles and wash out the free dye particles ( fig. 2.9 a) ). Then when time goes by free particles diffuse through the membrane to the outside. In ( fig. 2.9 b), c) ) you can see changes in the FCS profile. ( fig. 2.9 c) ) almost all free particles are outside the vesicles. Observing changes in the FCS profile we can evaluate the relative populations of slow and fast components and estimate the kinetics of the 
a)

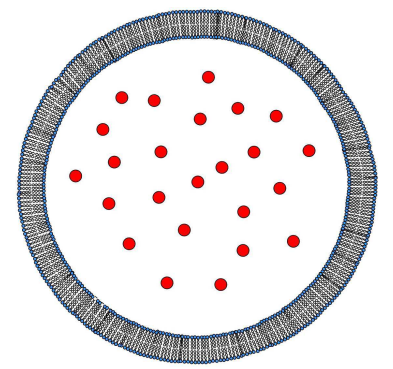

b)

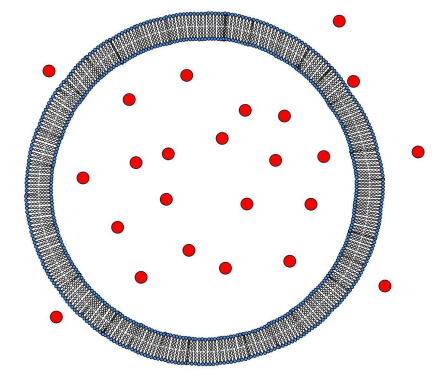

Е
C)

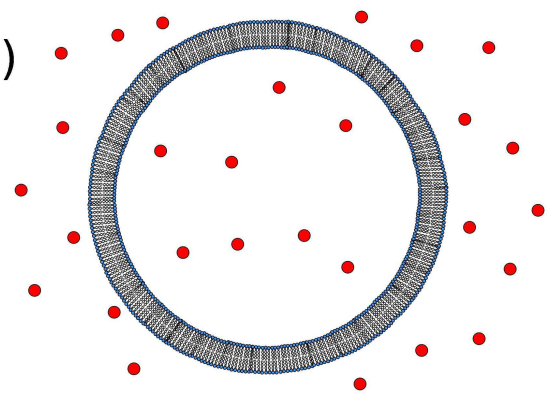

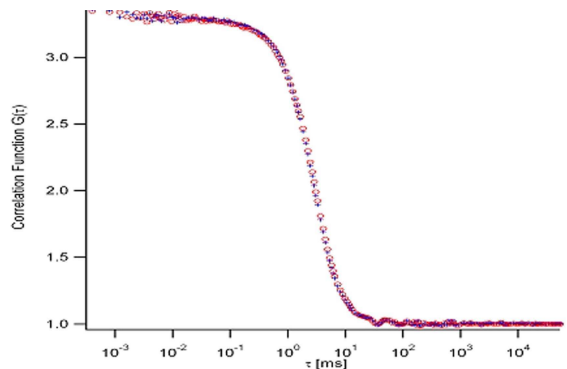
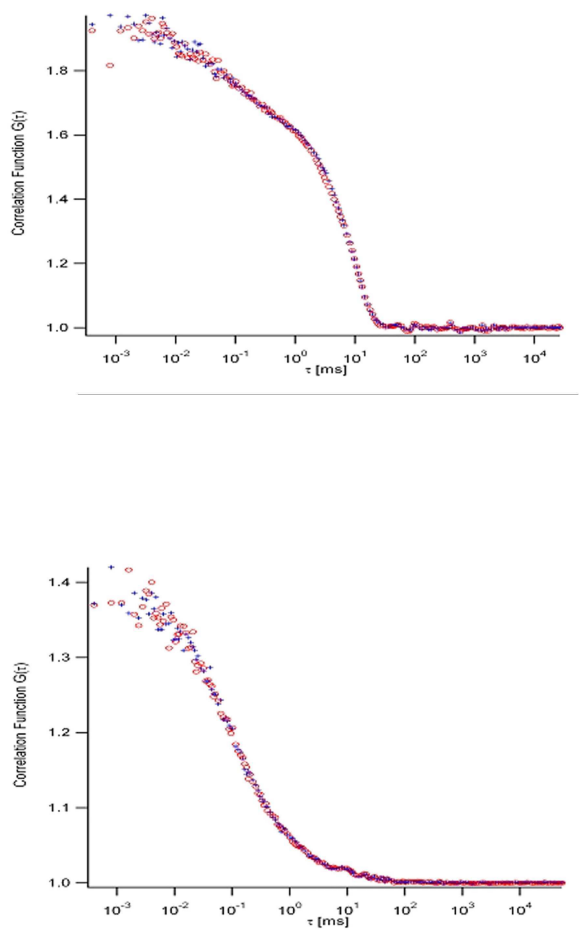

Figure 2.9: Permeation experiment. First I put dye particles inside the vesicles and wash out the free dye particles outside a). Then when time goes by free particles diffuse through the membrane to the outside. In b), c) you can see changes in the FCS profile.

permeation process.

When I measure the permeability dependence on temperature I repeat this 
procedure for different temperatures.

To estimate the relative populations of slow and fast components we use a two component correlation function formula for 3D from (Rigler and Elson, 2001), which is shown in equation (2.5).

The calibration of the experiment as it was described before justifies the use of this two-component fit formula for the estimation of the relative populations of slow and fast components, assuming no bleaching and dye absorption on the walls of the measurement cell.

It is also possible to use a formula for slow chemical reactions to describe the permeation process, but both formulae do not include the quenching influence and the dye absorption on the cell walls.

The exponential fit $y=A \exp (-b x)$, like it is presented in ( fig. 2.8) is used to obtain the coefficient $b$, which characterizes the permeability at each temperature. A parameter inverse to this coefficient the permeation time $\tau=2.3 / b$ can be introduced. The time $\tau=2.3 / b$ corresponds to the time when no slow component is any more detectable. The factor 2.3 describes the sensitivity of our FCS method. This permeation time can be intuitively understood as the time, when almost all dye particles are outside the vesicles and this is only reason for introducing this term and the factor 2.3. The time $\tau=1 / b$ gives a sufficient description of the permeation kinetics, but cannot be expressed in intuitively understandable terms. 

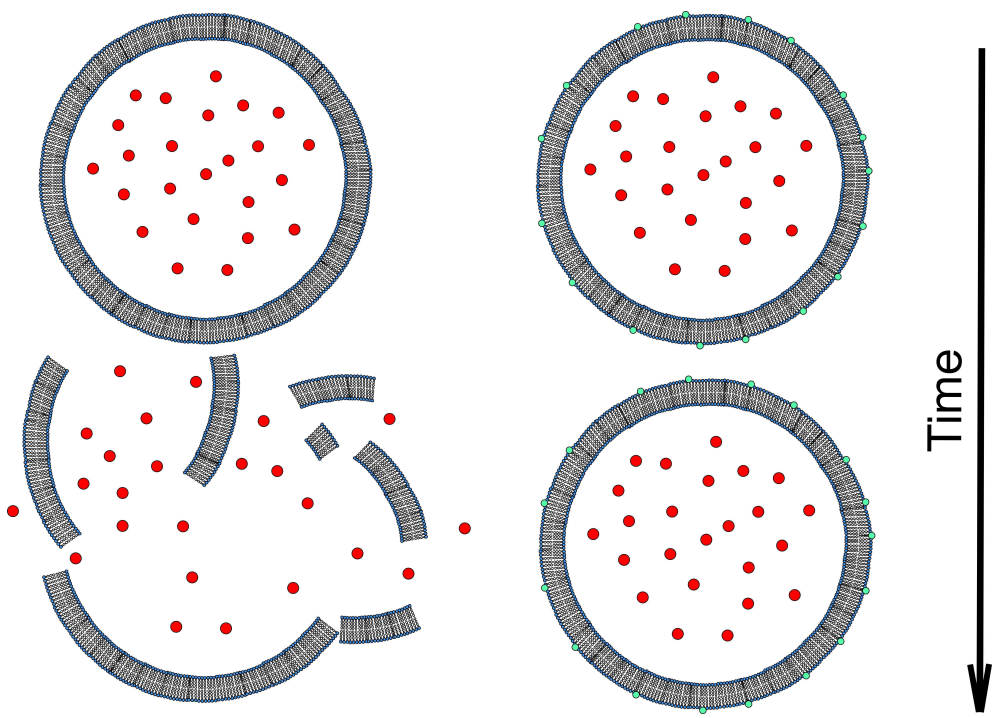

Figure 2.10: Adding of a small amout of charged lipid prevents fusion and makes the vesicles stabile.

\subsection{Probe preparation for Fluorescence Cor- relation spectroscopy}

First $10 \mathrm{mM}$ lipid solution DPPC + 5.0-7.5mol\% DPPG were prepared and mixed with Rhodamine 6G dye purchased from Molecular Probes Inc. ( www.probes.com ). Lipids were purchased from Avanti Polar Lipids (Birmingham, AL). 5.0-7.5\% of charged lipid DPPG was added with the aim to prevent fusion and breaking of vesicles ( fig. 2.10). DPPG has the same chain length and the same melting temperature as DPPC. Then unilamellar vesicles with dye particles inside were obtained using an extrusion process ( as described in the probe preparation section for calorimetry ). Dyes outside the vesicles were washed out using chromatography with Sephadex 75 gel purchased from Fluka (Buchs, Switzerland) after the extrusion process.

The chromatography procedure is described in more detail in the appendix 
A.

Lipid-peptide mixtures were first dissolved in organic solvent, then after evaporation put into a dessicator for 10-12 hours. 


\section{Chapter 3}

\section{Simulation approach}

\subsection{Ising-like model on a hexagonal lattice}

As a theoretical approach we use Monte-Carlo simulations. We use an Isinglike model on a hexagonal lattice. In our model a lipid can be in two states : so called gel and fluid states, like spin up and spin down in the Ising model ( fig. 3.1 ). We put lipids on a hexagonal lattice, so each lipid has 6 nearest neighbors and interacts only with these nearest neighbors.
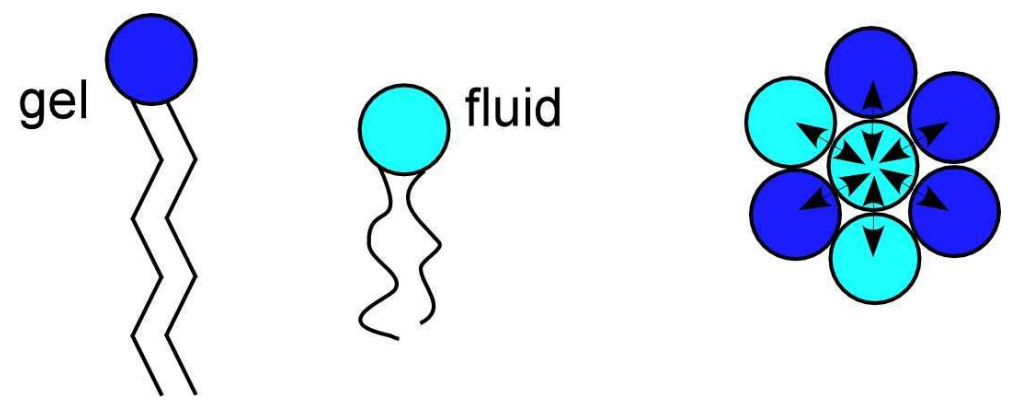

Figure 3.1: A lipid can be in two states : so called gel and fluid states, like spin up and spin down in the Ising model. We put lipids on a hexagonal lattice, so that each lipid has 6 nearest neighbors and interacts only with these nearest neighbors.

We put the lipids on triangular matrix and use periodic boundary conditions, 
therefore the matrix is topologically equivalent to a torus.

The free energy of each lipid consists of the intrinsic free energy of chain configuration $G$ and the sum of interaction energies with nearest neighbors $\varepsilon$. These interactions are temperature independent. This assumption is justified, as long as the half-width of the transition is small (Heimburg and Biltonen, 1996).

The total matrix energy is the sum of energies of all lipids :

$$
G=n_{g} \cdot G_{g}+n_{f} \cdot G_{f}+n_{g g} \cdot \varepsilon_{g g}+n_{f f} \cdot \varepsilon_{f f}+n_{g f} \cdot \varepsilon_{g f}
$$

were $n_{i}$ is the number of lipids in gel or fluid state $(i=g$ (gel) or $i=f$ (fluid) ), $n_{i j}$ is the number of nearest neighbor interactions of the lipids in states $i$ and $j$.

In the case of periodic boundary conditions :

$$
\begin{aligned}
& n_{f f}=\frac{z \cdot n_{f}-n_{g f}}{2} \\
& n_{g g}=\frac{z \cdot n_{g}-n_{g f}}{2}
\end{aligned}
$$

where $z=6$ is the coordination number.

Then free energy of the system :

$$
G=n \cdot G_{g}+n_{f} \cdot(\Delta H-T \cdot \Delta S)+n_{g f} \cdot \omega_{g f}
$$

where $\Delta H=\left(H_{f}+z \cdot \varepsilon_{f f} / 2\right)+\left(H_{g}+z \cdot \varepsilon_{g g} / 2\right), \Delta S=\left(S_{f}-S_{g}\right)$ and $\omega_{g f}=$ $\varepsilon_{g f}-\left(\varepsilon_{g g}+\varepsilon_{f f}\right) / 2$

The system has an energy minimum when all lipids are in the gel state. Then the excess free energy is :

$$
\Delta G=G-n \cdot G_{g}=n_{f} \cdot(\Delta H-T \cdot \Delta S)+n_{g f} \cdot \omega_{g f}
$$


Here $(\Delta H-T \cdot \Delta S)$ acts as a temperature -dependent field, and the model can be considered as an Ising model in an external field. However, there is no exact analogy because in the classical ferromagnetic Ising model external field does not depend on temperature. In this model the phase transition is totally field-induced.

The excess free energy $\Delta G=\left(\Delta H-T_{m} \cdot \Delta S\right)$ is equal to zero in the transition midpoint $T_{m}$ and therefore $\Delta S=\Delta H / T_{m}$ (Doniach, 1978).

In this model we have 3 parameters: enthalpy $\Delta H$, transition temperature $T_{m}$ and the cooperativity parameter $\omega_{g f}$, and all of them are obtained from experimental $\mathrm{Cp}$ profile. The enthalpy is the integral of the heat capacity profile over the temperature, $T_{m}$ is midpoint of the transition and the cooperativity parameter corresponds to the half-width of the transition and is obtained from fitting of the experimental curve.

\subsection{Heat capacity calculations and Glauber algorhitm}

Using the Monte-Carlo method we generate thermal fluctuations on the lipid matrix. Then we use the fluctuation-dissipation theorem (Hill, 1960) to calculate the heat capacity of the system :

$$
C_{p}=\frac{\left\langle H^{2}\right\rangle-\langle H\rangle^{2}}{R T^{2}}
$$

A Monte Carlo cycle simulates the thermal fluctuation of the system for a very short time. The values $\left\langle H^{2}\right\rangle$ and $\langle H\rangle$ are averaged over time (many MC cycles, time in Monte Carlo simulation has only meaning of a sequence of states). 
We use Glauber's algorhitm (Glauber, 1963) which has the following steps :

- Pick a random lattice point

- Change its state

- Calculate the Gibbs energy difference between the new and the old matrix configuration 3.2

$$
\delta G= \pm(\Delta H-T \cdot \Delta S)+\Delta n_{g f} \cdot \omega_{g f}
$$

where $+/-$ stands for changing gel-fluid/fluid-gel and $\Delta n_{g f}$ is the increase or decrease of the nearest neighbor contacts.

- Calculate the probability for the change

$$
P=\frac{K(T)}{1+K(T)}, K(T)=\exp \left(-\frac{\delta G}{R T}\right)
$$

- Generate a random number $R A N$ and compare it with the calculated probability.

- Make a decision ( $Y E S$ or $N O$ ) for the change.

$$
\begin{array}{r}
R A N \leq P \mapsto Y E S \\
R A N>P \mapsto N O
\end{array}
$$

After each Monte Carlo cycle the enthalpy is calculated : $H=n_{f} \cdot(\Delta H-$ $T \cdot \Delta S)+n_{g f} \cdot \omega_{g f}$. Average values $\langle H\rangle$ and $\left\langle H^{2}\right\rangle$ are calculated in order to determine the heat capacity. This procedure is repeated for different temperatures. 


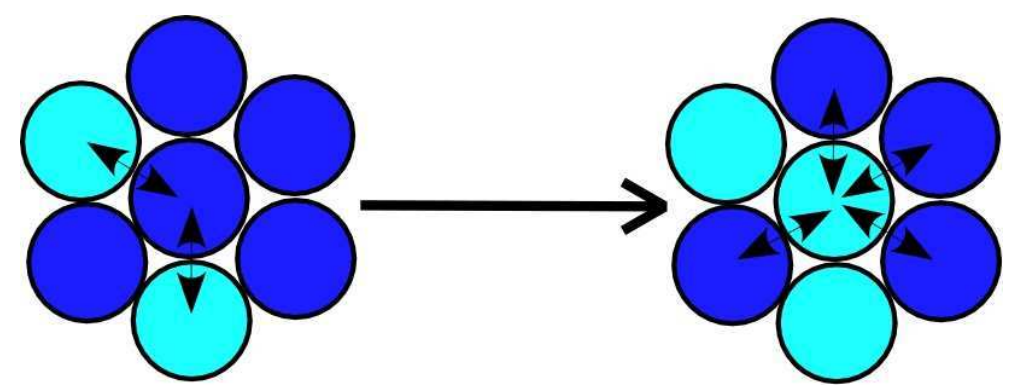

Figure 3.2: Illustration of $M C$ step. $\Delta n_{g f}=2, \delta G=(\Delta H-T \dot{\Delta} S)+2 \cdot \omega_{g f}$.

\subsection{Peptides}

In our model a peptide can occupy one or four places in the lattice. Gramicidin A has a size similar to four places in the lattice.

a)
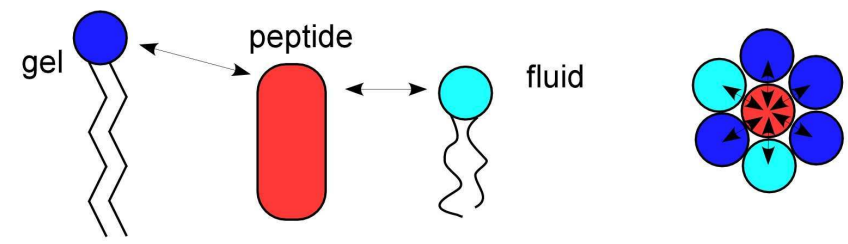

b)
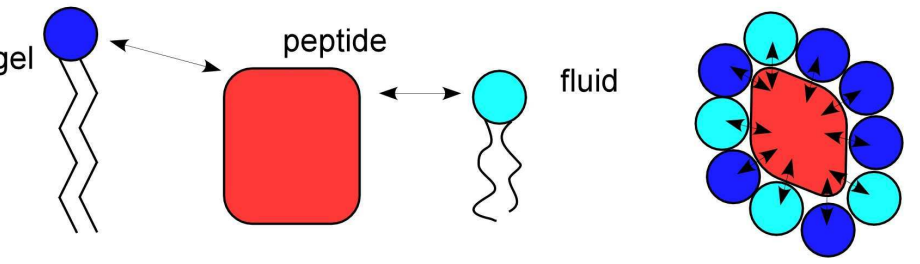

Figure 3.3: Illustration of lipid-peptide interactions. A peptide can occupy one a) or four b) places in the lattice and interacts with a different number of lipids.

We introduce two parameters to describe the peptide interactions with gel 
and fluid ( fig. 3.3 ):

$$
\begin{gathered}
\omega_{g p}=\varepsilon_{g p}-\left(\varepsilon_{g g}-\varepsilon_{p p}\right) / 2 \\
\omega_{f p}=\varepsilon_{f p}-\left(\varepsilon_{f f}-\varepsilon_{p p}\right) / 2
\end{gathered}
$$

Then the excess free energy (3.5) :

$$
\begin{array}{r}
\Delta G=n_{f} \cdot(\Delta H-T \cdot \Delta S)+n_{g f} \cdot \omega_{g f} \\
+n_{g p} \cdot \omega_{g p}+n_{f p} \cdot \omega_{f p}
\end{array}
$$

Used Monte Carlo algorithm :

- Pick at random a lattice point

- If it is a lipid, try to change its state ( proceed like described in section $3.2)$

- If it is a peptide :

- Pick one or four lipids at random.

- Exchange it with the peptide.

- Calculate the Gibbs energy difference between the new and the old matrix configuration

$$
\delta G=\delta G^{l i p i d}+\delta G^{\text {peptide }}
$$

where $\delta G^{l i p i d}=\left(\delta n_{g f}^{l i p i d} \cdot \omega_{g f}+\delta n_{g p}^{l i p i d} \cdot \omega_{g p}+\delta n_{f p}^{l i p i d} \cdot \omega_{f p}\right)$ and $\delta n_{g f, g p, f p}^{l i p i d}$ is the increase unlike near neighbor contacts of the lipid. $\delta G^{\text {peptide }}$ is given by a similar expression. 
- Calculate the probability for the change

$$
P=\frac{K(T)}{1+K(T)}, K(T)=\exp \left(-\frac{\delta G}{R T}\right)
$$

- Generate a random number $R A N$ and compare it with the calculated probability.

- Make a decision ( YES or NO) for the exchange of the lipid and peptide.

$$
\begin{array}{r}
R A N \leq P \mapsto Y E S \\
R A N>P \mapsto N O
\end{array}
$$

The introduction of the peptides in the model leads to increasing of number of needed parameters on 2: $\omega_{g p}$ and $\omega_{f p}$.

\subsection{Introduction of compressibility into the model and permeability simulation}

To simulate the membrane permeability we need to introduce compressibility into the model. We also want to relate spontaneous pore formation processes to local lipid fluctuations. Lipid fluctuations have a meaning of area fluctuations first of all. The area difference between a lipid in a gel and fluid state is about $25 \%$.

If one takes four lipids being in a fluid state, one change three of them to a gel lipid and makes a pore which size is equal to the size of a lipid in the gel state, removing one fluid lipid to the edge of the matrix ( fig. 3.4).

Let us assign to a lipid in fluid state an area, equal to 4 area units. Then the area, assigned to a lipid in the gel state would be 3 area units, because the difference between the lipid area in the fluid and the gel state is about $25 \%$. 

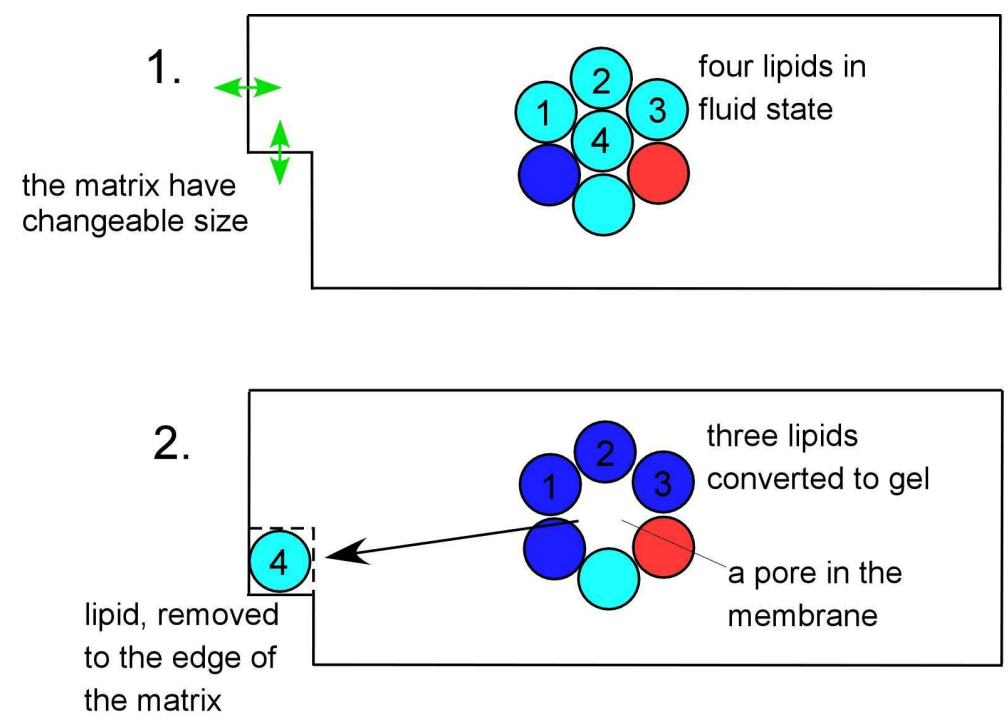

Figure 3.4: Illustration of the way to introduce compressibility into the model. Three fluid lipids is converted to gel and one central fluid lipid is removed to the edge of the matrix. In his place a pore is made, which size is is equal to size of gel lipid. After such MC step total area of the membrane is the same.

Before a MC step the area which is occupied by 4 lipids in the fluid state is : $A_{\text {before }}=4 \cdot A_{f}=4 \cdot 4=16$.

The area after a MC step is $A_{\text {after }}=3 \cdot A_{g}+A_{h}+A_{f}=3 \cdot 3+3+4=16$.

The total area of the membrane would remain the same after such a MC step.

Thus to make a pore in the membrane we need to compress it. This is an indirect way to introduce compressibility into the model. In order to make possible such a Monte Carlo step possible we developed a program, which operates with a matrix of changeable size, keeping quasi-periodic boundary conditions ( fig. 3.5).

Points, which are located on the colored strips inside the matrix are copied 


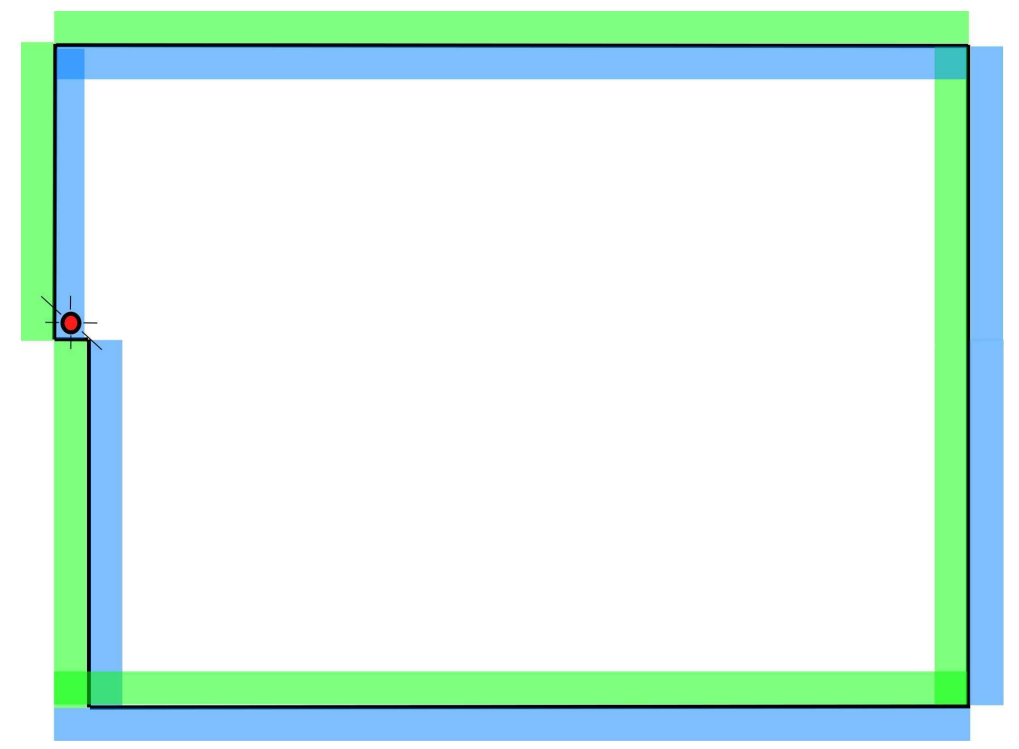

Figure 3.5: Schema of boundary conditions for our matrix of changeable size. Points, which are located on the colored strips inside the matrix are copied to the correspondent colored strip outside the opposite side of the matrix. The red circle represents the edge point on the matrix, where lipids, removed after creating a pore are inserted ( and lipids, needed to destroy a pore are taken from ). Black lines represent interactions with nearest neighbors ( 6 in case of hexagonal lattice). This number of point in the last row of the matrix (where the red point is) can change, while creating or destroying the pores. As well the number of rows can change, when the end this last row is reached by the red point.

to the correspondent colored strip outside the opposite side of the matrix. The red circle represents the edge point on the matrix, where lipids, are removed to after the creation of a pore ( and from where lipids are taken in order to destroy a pore ). Black lines represent interactions with nearest neighbors ( 6 in case of a hexagonal lattice ). The number of points in the last row of the matrix ( indicated through the red point) can change, while creating or destroying the pores. As well, the number of rows can change, when the end of this last row is reached by the red point. However, there are two special points in the matrix : insertion point, marked by red circle 
and corner point, where one of the nearest neighbours is different for the point in the matrix and its image on the strip, attached to the opposite edge. At these two points boundary conditions are not strictly periodic. However, the energy contribution from these two points for the whole matrix energy is small, and is different from case with strict periodic boundary conditions only if this neighbor place for this special point in the matrix and its image is not occupied by the same sort of lipid, or peptide or hole, which occurs only seldom because of the presence of domains. This schema of boundary conditions allows us to keep unchanged topology of the matrix and therefore, the domain structure and peptide aggregates when creating and destroying pores. It is not possible, when using a matrix of fixed size, like in (Founier and Joos, 2003). Our schema of boundary conditions allows us to take into account the influence of local inhomogeneities and therefore processes at domain borders and near peptide aggregates, in which we are interested in. This is the main reason for developing simulation approach, which makes use of a matrix of changeable size and proposed boundaries.

This is the way we create the matrix of changeable size with quasi - periodic boundary conditions.

To describe pore interactions with lipids in fluid and gel states and peptides we need to introduce three other parameters to the model : $\omega_{g l}, \omega_{f l}$ and $\omega_{p l}$ for gel-pore, fluid-pore and peptide-pore interactions respectively.

The used Monte Carlo algorithm can be briefly represented as follows:

- Pick randomly a point in the lattice

- If it is a fluid try to find three lipids in fluid state in the near neighborhood. 
- If there are three lipids in fluid state change their state to gel, and remove central lipid to the edge of the matrix.

- Calculate the Gibbs energy difference between the new and the old matrix configuration 3.4

$$
\delta G=-3 \cdot(\Delta H-T \cdot \Delta S)+\sum \Delta n_{i j} \cdot \omega_{i j}
$$

where -3 stands for the change fluid-gel for three lipids and $\Delta n_{i j}$ is the increase of the nearest neighbor contacts, $i, j$ for gel, fluid, peptide or pore.

- Calculate the probability for the change

$$
P=\frac{K(T)}{1+K(T)}, K(T)=\exp \left(-\frac{\delta G}{R T}\right)
$$

- Generate a random number $R A N$ and compare it with the calculated probability.

- Make a decision ( $Y E S$ or $N O$ ) for the change.

$$
\begin{gathered}
R A N \leq P \mapsto Y E S \\
R A N>P \mapsto N O
\end{gathered}
$$

There are also steps included in which two lipids melt and a pore with size of $2 / 3$ of the size of a lipid in gel state is created, and one lipid is melted and a pore with size of $1 / 3$ of the size of a lipid in gel state is created.

Also reverse steps, which means the destruction of a pore are included to satisfy detailed balance. As well thre are steps included which make pore diffusion exactly as it was described for peptides in the previous section. 
The realization of lipid melting and peptide diffusion stays the same as described in sections $3.1,3.2$.

The area, occupied by pores is averaged over many MC cycles for each temperature and the dependence area, occupied by pores on temperature is build. The calculations were done with a matrix of size $100 \times 200$. This reduces the influence of the boundary in comparison with smaller matrices.

The use of the matrix of changeable size with periodic boundary conditions allows us to study pore formation processes without disturbing the topology of the system.

This procedure allows us to relate spontaneous pore formation processes to local lipid fluctuations. This model takes into account the influence of local heterogeneities of the lipid bilayer ( like domain border, peptides ) on membrane permeability. 


\section{Chapter 4}

\section{Results}

\subsection{Gramicidin A aggregation study : calorime- try results}

Calorimetric measurements were done on unilamellar pure DPPC vesicles, and with addition of $1.25 \mathrm{~mol} \%$ and $2.5 \mathrm{~mol} \%$ of Gramicidin A ( fig. 4.1 ).

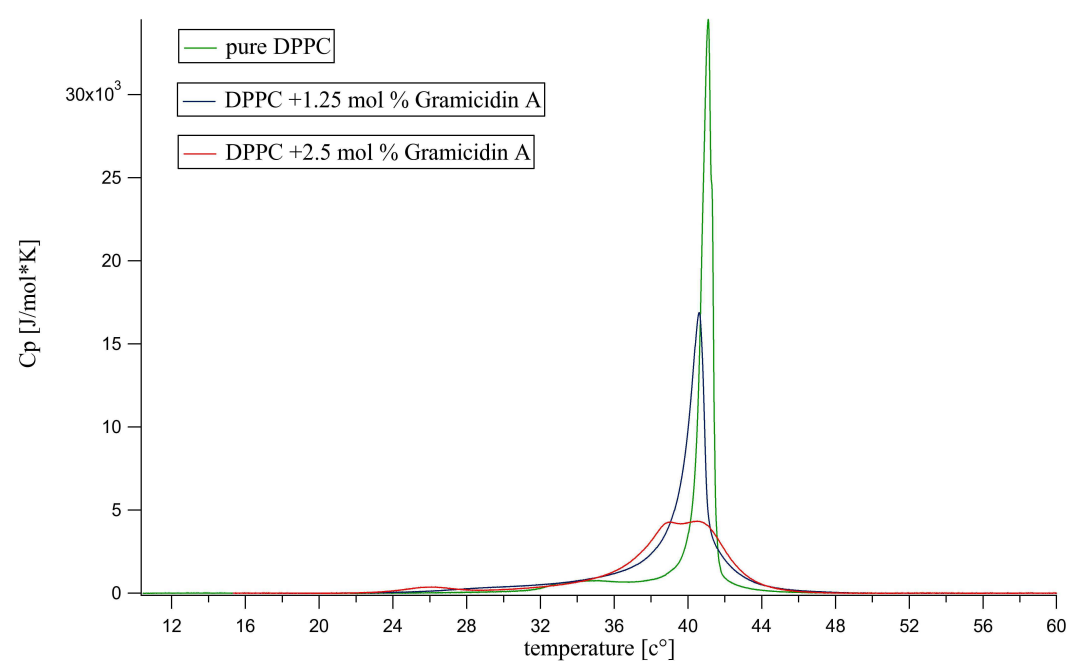

Figure 4.1: Experimental heat capacity profile of unilamellar pure DPPC vesicles, and with addition of 1.25 mol\% and 2.5 mol\% of Gramicidin A. 
Gramicidin A is a small peptide, which forms head to head dimers (Andersen et al., 1999). NMR studies of the structure of Gramicidin A were done by Ketchem (Ketchem et al., 1993). Gramicidin A is relatively short $2.6 \mathrm{~nm}$ and shorter as both gel and fluid phases of DPPC membranes (between 3.5 and $4.8 \mathrm{~nm}$ ). Addition of Gramicidin A shifts the maximum of heat capacity profile down to lower temperatures and broadens the profile.

\subsection{Gramicidin A aggregation study : Monte Carlo results}

Monte Carlo simulations were done with the aim to understand the aggregation behavior of Gramicidin A from measured heat capacity profiles. Fig. 4.2 shows the fitting of experimental heat capacity profiles for pure DPPC and DPPC $+1.25 \mathrm{~mol} \%$ Gramicidin A unilamellar lipid vesicles. In the simulations a peptide can occupy one or four lattice points.

The red solid lines represent calorimetric curves, and the dashed or dotted lines represent theoretical profiles. The graph on the top corresponds to the pure lipid system. The shown parameters are obtained from the fitting and are used in the simulations with peptide. The central picture of fig. 4.2 shows Monte Carlo simulations with 1-site peptides and $5 \%$ of all points in the matrix are occupied by peptide, which corresponds to the area, which are occupied by Gramicidin A in experiments. To demonstrate the uniqueness of the best theoretical profile, two more profiles with the same shift of heat capacity maximum but different absolute values of $\omega_{g p}$ and $\omega_{f p}$ are given by blue and green dashed/dotted lines. The bottom picture shows simulation with a peptide, occupying 4 sites in the lattice and $10 \%$ of all lattice sites 

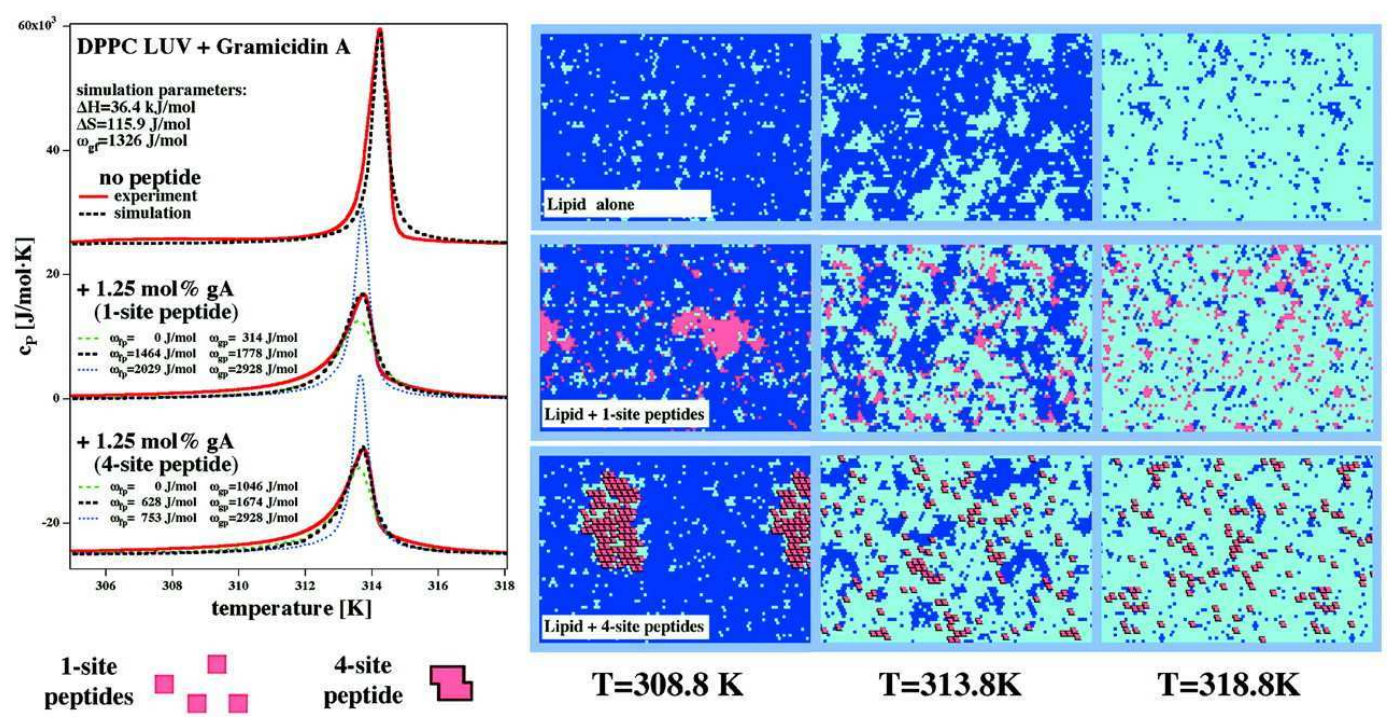

Figure 4.2: Fitting of experimental heat capacity profiles for pure DPPC and DPPC +1.25 mol\% Gramicidin A unilamellar vesicles. In the simulations a peptide occupies one or four places in the lattice. MC snapshots for three different temperatures : below and above the phase transition point and in the transition regime. Domains of gel ( solid-ordered) and fluid (liquid-disordered) are represented by dark blue and bright blue colors. Peptides are presented by red color. (Picture taken from (Ivanova et al., 2003)).

are occupied by peptide, corresponding to $2.5 \mathrm{~mol} \%$ of peptide. The right part of the picture shows snapshots from MC simulation at three different temperatures, below the transition temperature, in the transition regime and above the transition temperature. The three pictures at the top show simulations with only lipid, the center three snapshots show simulation with $10 \%$ of the lattice sites occupied by 1-site peptides, and the three bottom pictures show simulation result with $2.5 \%$ of peptide, which occupies 4 sites. Gramicidin A shows a preference to the fluid phase, which corresponds to a shift of the maximum of heat capacity profile to lower temperatures. A peptide, which has the preference to gel phase would shift the $\mathrm{Cp}$ maximum to higher temperatures, and a peptide, which interacts equally with both phases 
would not shift the heat capacity maximum at all, but would still result in boardening of the profiles. In the case of the Gramicidin A the shift to lower temperatures is reasonable, because the hydrophobic core of Gramicidin A is smaller that the lipid bilayer thickness in the fluid phase and is significantly smaller then the lipid bilayer thickness in the gel phase. This result therefore supports the concept of "hydrophobic matching" (Mouritsen and Bloom, 1984).

Snapshots show that Gramicidin A forms aggregates in both gel and fluid phases. The cluster sizes are larger in the gel than the fluid phase. Close to peptide clusters lipids are more likely to be in a fluid state. This is a consequence of $\omega_{g p}$ being larger then $\omega_{f p}$.

Simulation with modeling the peptide on 1-site have the disadvantage that we take four peptides, each $1 \mathrm{x}$ site instead of one peptide $4 \mathrm{x}$ site for simulate gramicidin A. This leads to overestimation of mixing entropy of the peptides and underestimating their tendency to aggregate. To estimate the influence of this was the main aim of the simulation with $4 \mathrm{x}$ site peptides.

The simulated heat capacity profiles and the aggregation patterns are quite similar for simulations with 1-site and 4-site peptide. However, the exact values of the interaction parameters $\omega_{g p}$ and $\omega_{f p}$ are different. This means that the heat capacity profiles are rather influenced by the nature of mixing behavior than dominated by the exact values of the interaction parameters. Therefore we have similar Monte Carlo snapshots and similar degrees of aggregation in both models. 


\subsection{Gramicidin A aggregation study : Local Fluctuations}

The heat capacity is proportional to enthalpy fluctuations on the whole matrix(3.6). Other response functions are, as well, proportional to fluctuations. The isothermal compressibility can be derived from volume fluctuations $\left(\kappa_{T}=\left(\left\langle V^{2}\right\rangle-\langle V\rangle^{2}\right) / V \cdot R T\right)$ (Heimburg, 1998). Bending elasticity (Heimburg, 1998),(Heimburg, 2000) and relaxation times after small temperature or volume perturbations (Grabitz et al., 2002) are also proportional to fluctuations.

One can see from snapshots ( fig. 4.2 ), that the lipid and peptide distributions are obviously not uniform, and one can ask the question whether response functions are uniform or whether they reflect the domain structure of the lipid - peptide matrix. Simulations of local fluctuations were done with aim to address this question.

Local fluctuations were calculated for each lipid on the matrix.

We assign values $S_{i j}=0$ for the gel state and $S_{i j}=1$ for the fluid state. We can calculate for each lipid the mean probability to be in the fluid state $0 \leq S_{i j} \leq 1$ and the fluctuations around this average. Fluctuations have a maximum at the melting point with equal probability to be in gel or fluid state $\left\langle S_{i j}\right\rangle=0.5$ and $\left\langle S_{i j}^{2}\right\rangle=0.5$. So we can define :

local fluctuations $\equiv\left\langle S_{i j}^{2}\right\rangle-\left\langle S_{i j}\right\rangle^{2}$

with

local fluctuations $=0$ pure gel state

local fluctuations $=0.25$ at melting point 


\section{MC snapshots MC snapshots} (averaged)

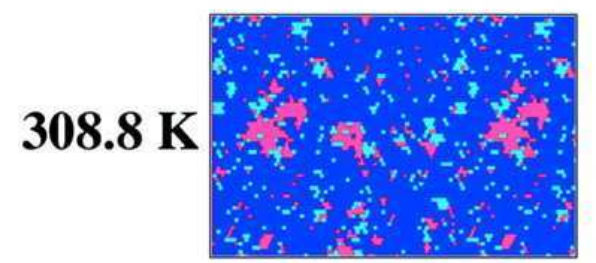

$311.3 \mathrm{~K}$

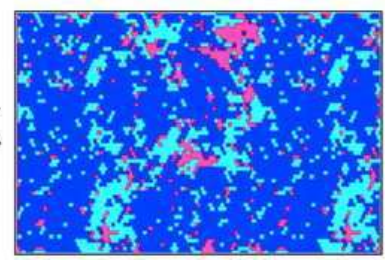

$313.8 \mathrm{~K}$

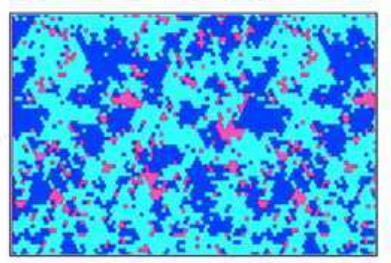

$316.3 \mathrm{~K}$

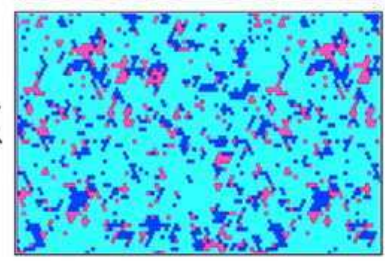

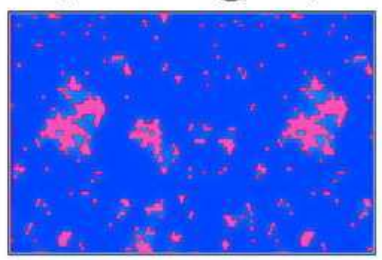
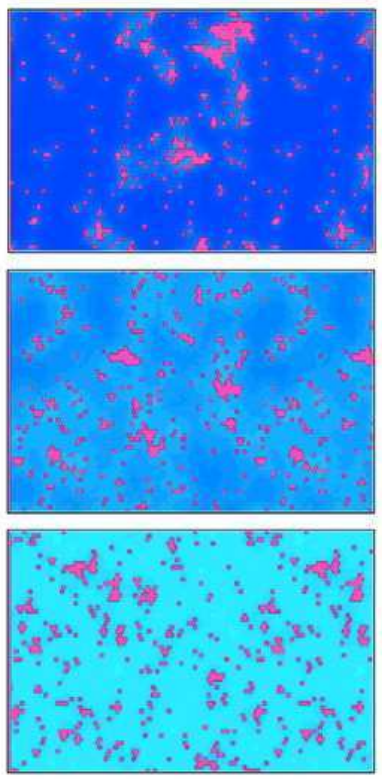

\section{local fluctuations}
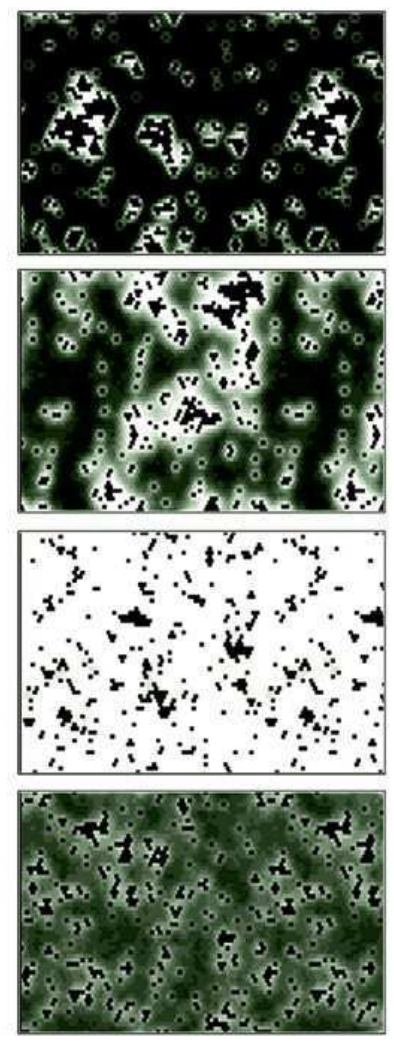

Figure 4.3: Monte Carlo snapshots ( simulation of Gramicidin A-containing membrane, $1 x$ site peptide) $S_{i j}$ - left column (Domains of gel (solid-ordered) and fluid ( liquid - disordered) are presented by dark blue and bright blue correspondingly. Peptides are presented by red color), averaged lipid state $\left\langle S_{i j}\right\rangle$ - central column, local fluctuations $\left\langle S_{i j}^{2}\right\rangle-\left\langle S_{i j}\right\rangle^{2}$ - right column. Brighter shadows mean larger fluctuations. The color scale varies between 0.0 - black and 0.25 white. (Picture taken from (Ivanova et al., 2003)).

local fluctuations $=0$ pure fluid state

First, we equilibrate the system, then we switch off the peptide diffusion, and we continue simulation for a given peptide - lipid configuration and calculate the local fluctuations as defined above. ( such definition would be correct 
only for a system where the peptides do not diffuse. )

Now, we can plot the local fluctuations as a function of lateral coordinates ( fig. 4.3 ).

Here representative Monte Carlo snapshots ( simulation of Gramicidin A - containing membrane, $1 \mathrm{x}$ site peptide ) are shown ( $S_{i j}$ - left column, averaged lipid state $\left\langle S_{i j}\right\rangle$ - central column, local fluctuations $\left\langle S_{i j}^{2}\right\rangle-\left\langle S_{i j}\right\rangle^{2}-$ right column ). Brighter shadows means larger fluctuations. The color scale varies between 0.0 - black and 0.25 - white.

The local fluctuations are larger near peptides.

However, one should mention that the fluctuations near the peptide are smaller, when compared to the fluctuations of the pure lipid membrane at the phase transition temperature of the pure lipid membrane.

Gramicidin A shifts the phase transition temperature to lower values, meaning that the probability to find a lipid in fluid state near a peptide is bigger that in gel one.

As the response functions are coupled to the fluctuations it must be concluded that, lateral compressibility, bending elasticity are strongly altered near peptides, because they are related to the magnitude of the fluctuations. Therefore, membrane permeability should be altered near the peptide, because the probability of spontaneous pore formation should be related to lateral compressibility.

The membrane permeability should be increased near the peptide at the temperatures where heat capacity of membrane, containing peptide is higher as heat capacity for pure lipid membrane, like in the temperature range below main main phase transition for the lipid membrane containing Gramicidin 
A, see ( fig. 4.1 ). And the membrane permeability sould be smaller at the temperatures, where heat capacity for pure lipid membrane is higher, like the main phase transition temperature for the pure lipid membrane.

\subsection{Membrane permeability : FCS results}

The membrane permeability dependence on temperature was measured for a pure lipid membrane and for a membrane, containing 2.5 mol \% ot Gramicidin A.

In fig. 4.4 the permeability dependence on temperature is plotted for a pure lipid system.

The permeation time can be understood as the time when almost all dye particles are outside the vesicles and is obtained from fitting for each temperature (see experiment section for details). At the top the corresponding heat capacity profile is shown.

The permeability shows a maximum at the phase transition point. The permeability is lower below the transition point and higher above it. Similar results were observed in measurements (Papahadjopoulos et al., 1973) done with membranes, containing high concentration of cholesterol ( $30 \%-60 \%$ ) and a much lower temperature resolution. Such huge amounts of cholesterol remove the influence of cooperativity effects, we are interested in.

In our experiment a much better temperature resolution is achieved and no cholesterol was used with the aim to keep the system simple enough to be simulated by our MC approaches. However, at and above the transition temperature exactitude of permeation time measurement is not very high.

Fig. 4.5 shows the influence of Gramicidin A on membrane permeability. 

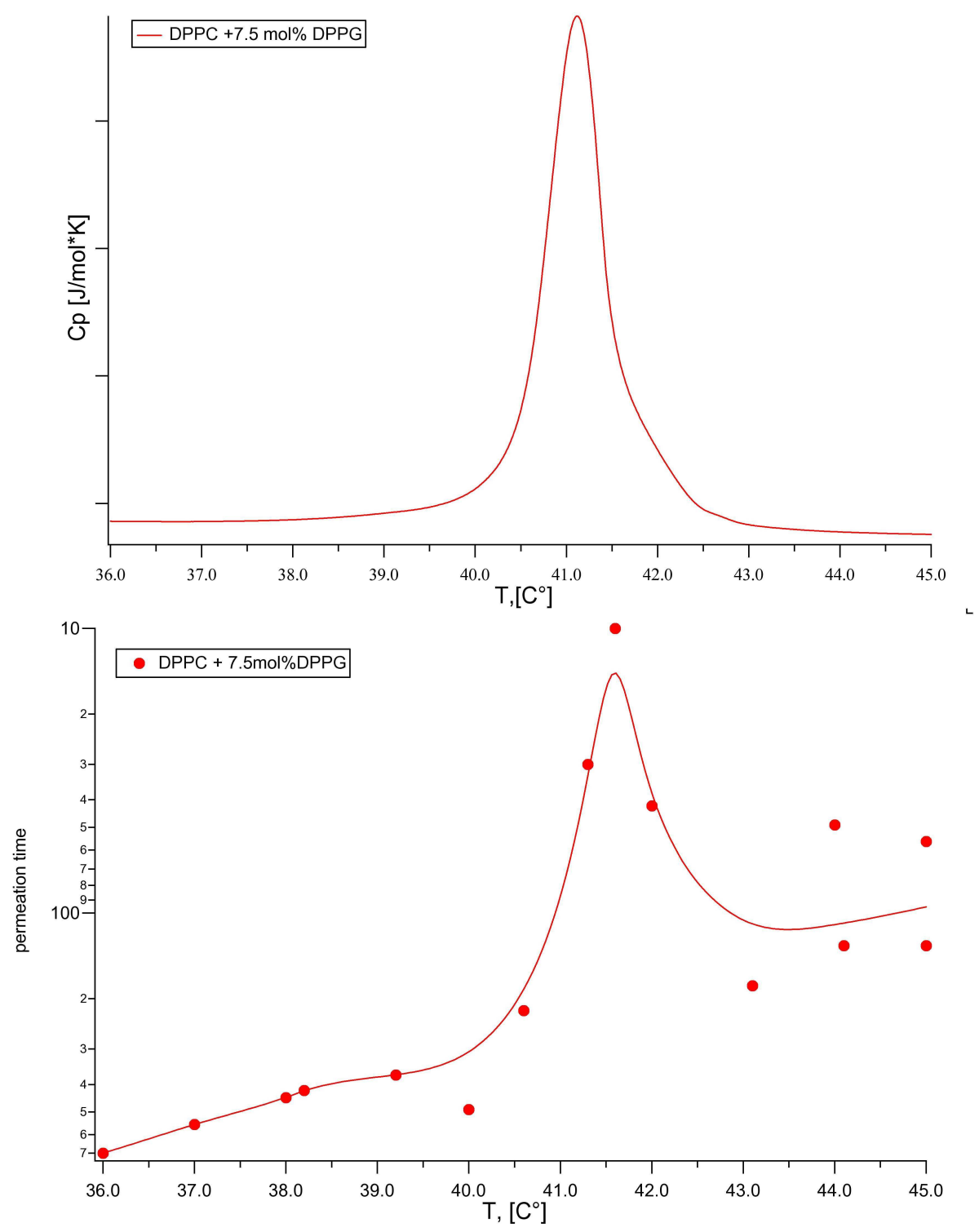

Figure 4.4: Permeability dependence on temperature for a pure lipid system : DPPC $+7.5 \mathrm{~mol} \%$ DPPG. Line serves as the guide to the eye. At the top the heat capacity profile is shown.

The red curve describes the permeability dependence on temperature for a pure lipid ( see also fig. 4.5) , blue one shows the permeability for a lipid membrane, containing $2.5 \%$ Gramicidin A. The top picture presents the 


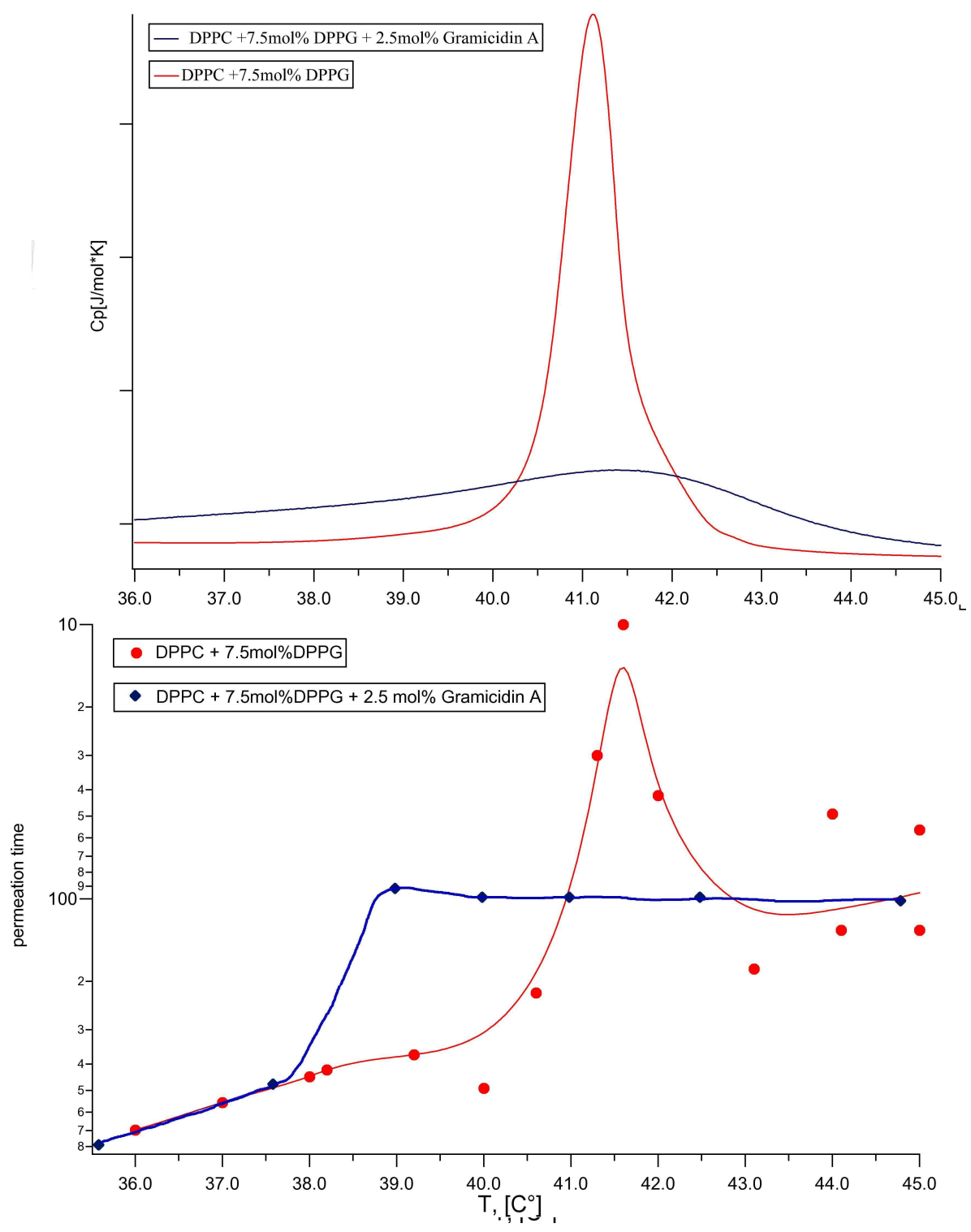

Figure 4.5: Permeability dependence on temperature for pure lipid membrane : $D P P C+7.5$ mol \% DPPG, and for a membrane with addition of 2.5 mol \% of Gramicidin A. Line serves as the guide to the eye. At the top the corresponding heat capacity profiles are shown.

corresponding heat capacity profiles.

Addition of Gramicidin A shifts the permeability maximum to lower tem- 
peratures. At the temperatures $35.5^{\circ}-37.5^{\circ}$ the profiles for the membrane without peptide and the membrane with peptide show similar values of permeability. By appr. $39^{\circ}$ the profile for the membrane with peptide has a maximum and high permeability values at $40^{\circ}-45^{\circ}$. the profile for pure lipid membrane have maximum at $41.3^{\circ}-41.6^{\circ}$. At the temperatures $38^{\circ}-$ $41^{\circ}$ the peptide containing membrane shows higher permeability as the pure lipid membrane. At $41^{\circ}-43^{\circ}$ the peptide containing membrane shows lower permeability in comparison to the pure lipid membrane.

Peptides change membrane permeability, influencing the local lipid environment.

With the aim to understand more about this influence a MC simulation approach was developed ( see results in the next section ).

\subsection{Membrane permeability : Monte Carlo results}

Fig. 4.6 presents simulation results for a pure lipid system.

In the case of the pure lipid system two parameters $\omega_{l g}$ and $\omega_{l f}$ describe the interactions between a pore and lipids in gel or fluid state respectively. The parameter $\omega_{g f}$, which describes the interactions between the lipids in gel and fluid state is taken from the previous study (Gramicidin A aggregation ) and is obtained there from the fitting of the experimental $\mathrm{Cp}$ profile for the pure lipid membrane (see section 4.2 for more details). Changing $\omega_{l g}$ and $\omega_{l f}$ parameters we can obtain a reasonable description of the permeability below and above the transition temperature (values of area, occupied by pores in gel and fluid states). In the phase transition point we have a maximum, in 


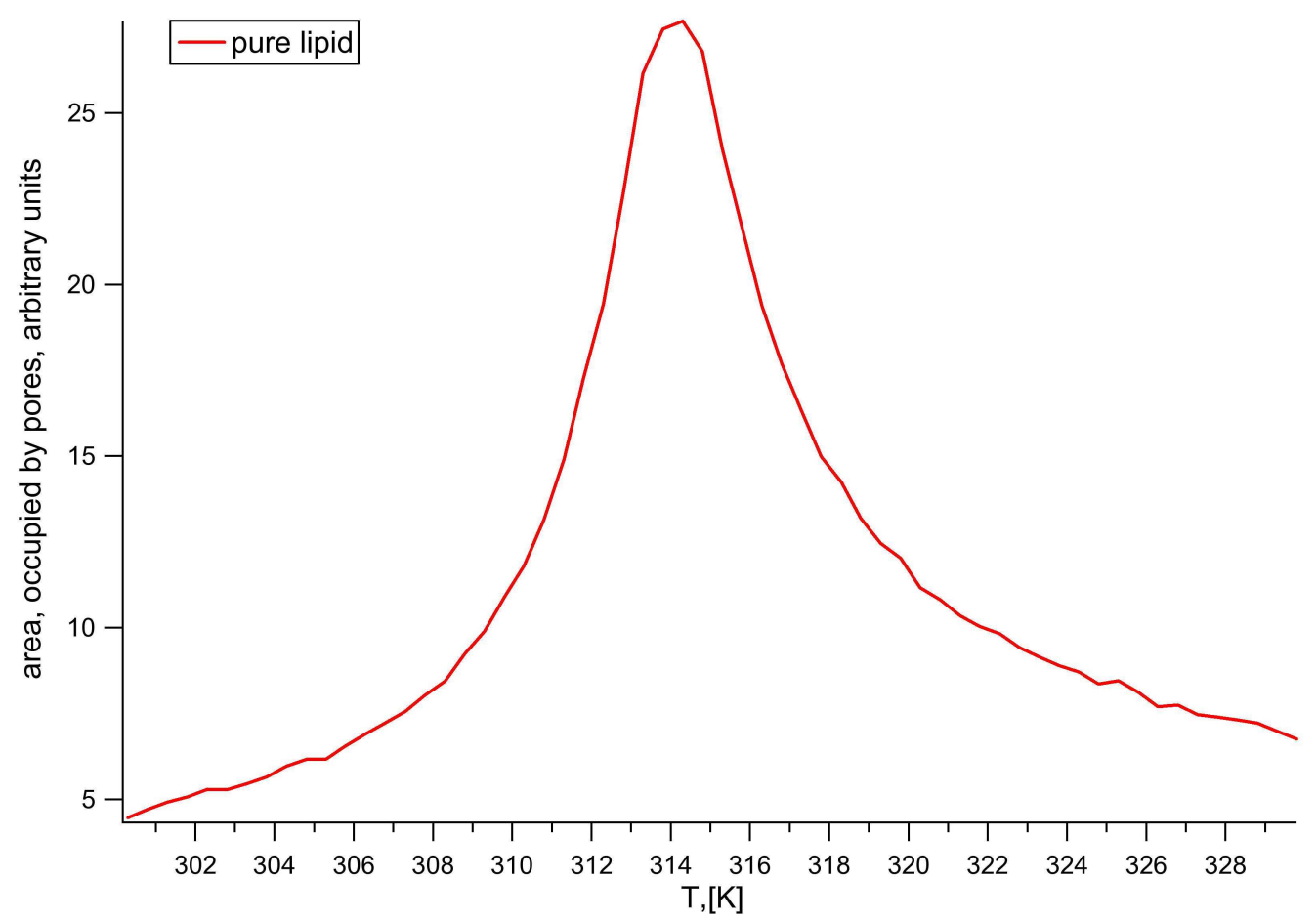

Figure 4.6: Simulation of permeability dependence on temperature for pure lipid membrane. The red curve represents the result of the simulation with $\omega_{l g}=2969$ $\mathrm{J} / \mathrm{mol}$ and $\omega_{l f}=522 \mathrm{~J} / \mathrm{mol}$.

agreement with experimental data ( fig. 4.4).

Let us consider two limiting cases to understand how these parameters influence the shape of the simulated permeability on temperature dependence ( fig. 4.7 ).

The red curve represents the result of a simulation with $\omega_{l g}=2969 \mathrm{~J} / \mathrm{mol}$ and $\omega_{l f}=522 \mathrm{~J} / \mathrm{mol}$. This curve is also shown in fig. 4.8. The blue one corresponds to a simulation with $\omega_{l g}=3346 \mathrm{~J} / \mathrm{mol}$ - larger than $\omega_{l g}$ for the red one and $\omega_{l f}=104 \mathrm{~J} / \mathrm{mol}$ - smaller than $\omega_{l f}$ for the red one. Such a change of the parameters results in a decrease in the area, occupied by pores in the gel phase and an increase in the fluid phase. The position of 


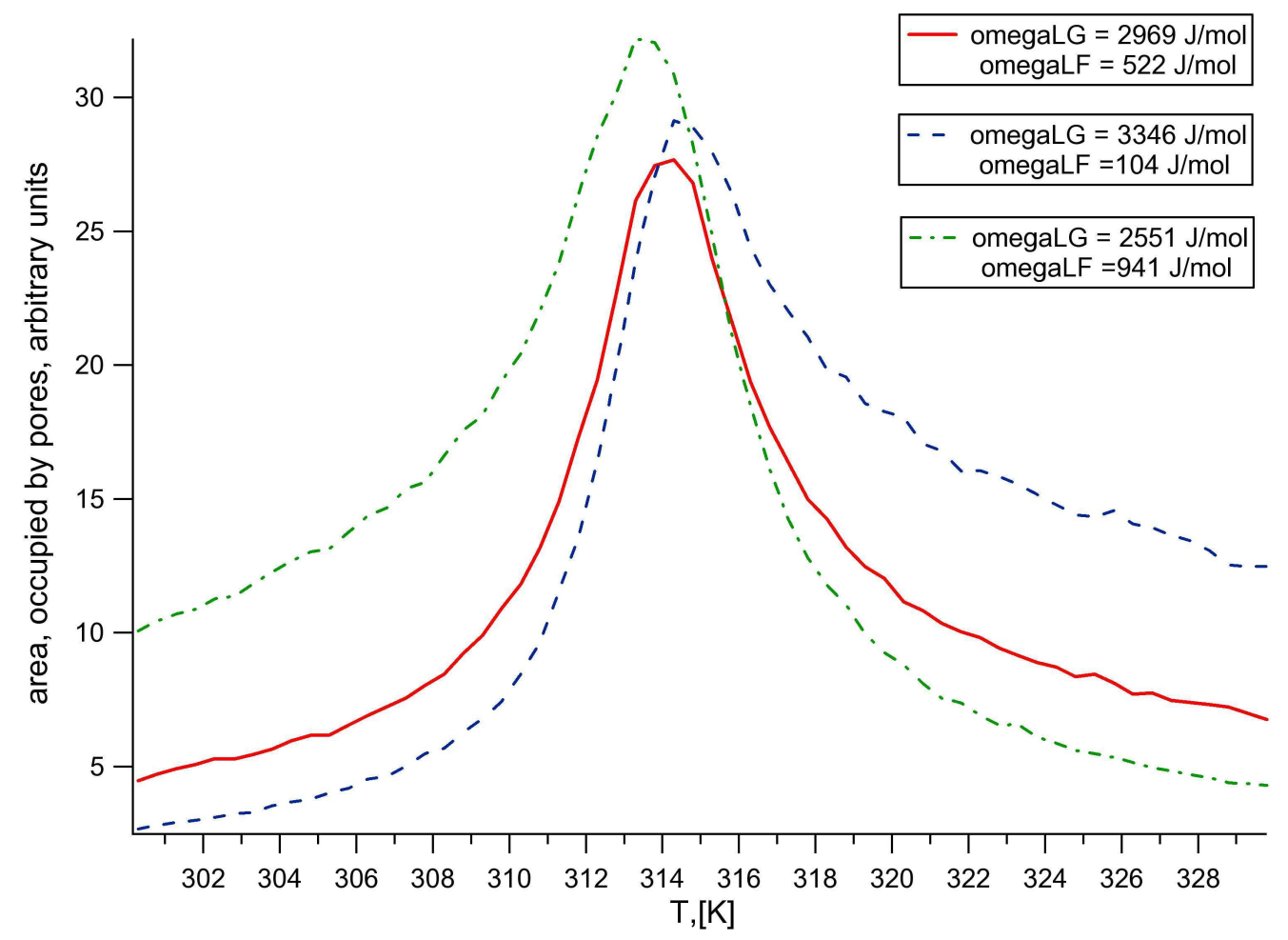

Figure 4.7: Results of MC simulation of permeabilty dependence on temperature for different sets of parameters. The red curve represents the result of a simulation with $\omega_{l g}=2969 \mathrm{~J} / \mathrm{mol}$ and $\omega_{l f}=522 \mathrm{~J} / \mathrm{mol}$. This curve is also shown in fig. 4.8. The blue one corresponds to a simulation with $\omega_{l g}=3346 \mathrm{~J} / \mathrm{mol}$ - larger than $\omega_{l g}$ for the red one and $\omega_{l f}=104 \mathrm{~J} / \mathrm{mol}$ - smaller than $\omega_{l f}$ for the red one. Such a change of the parameters results in a decrease in the area, occupied by pores in the gel phase and an increase in the fluid phase. The position of the maximum is slightly shifted to higher temperatures as well. The green one corresponds to a simulation with $\omega_{l g}=$ $2551 \mathrm{~J} / \mathrm{mol}$ - smaller then $\omega_{l g}$ for the red one and $\omega_{l f}=941 \mathrm{~J} / \mathrm{mol}$ - larger then $\omega_{l f}$ for the red one. Such a change of the parameters results in an increase in the area, occupied by pores in the gel phase and a decrease in the fluid phase. The position of the maximum is slightly shifted to lower temperatures. The values $\omega_{l g}=2969$ $\mathrm{J} / \mathrm{mol}$ and $\omega_{l f}=522 \mathrm{~J} / \mathrm{mol}$ in our simulation are chosen to fix the position of the maximum according to experimental result ( fig. 4.4 ), as well as the relative area, occupied by pores in the gel and fluid phases. The permeability of a fluid phase is higher, than the one in a gel phase.

the maximum is slightly shifted to higher temperatures as well. The green one corresponds to a simulation with $\omega_{l g}=2551 \mathrm{~J} / \mathrm{mol}$ - smaller then $\omega_{l g}$ 
for the red one and $\omega_{l f}=941 \mathrm{~J} / \mathrm{mol}$ - larger then $\omega_{l f}$ for the red one. Such a change of the parameters results in an increase in the area, occupied by pores in the gel phase and a decrease in the fluid phase. The position of the maximum is slightly shifted to lower temperatures. The values $\omega_{l g}=2969$ $\mathrm{J} / \mathrm{mol}$ and $\omega_{l f}=522 \mathrm{~J} / \mathrm{mol}$ in our simulation are chosen to fix the position of the maximum according to experimental result ( fig. 4.4 ), as well as the relative area occupied by pores in the gel and fluid phases. The permeability of a fluid phase is higher, than the one in a gel phase. This gives us quite reasonable agreement between the experimental result and the simulation result.

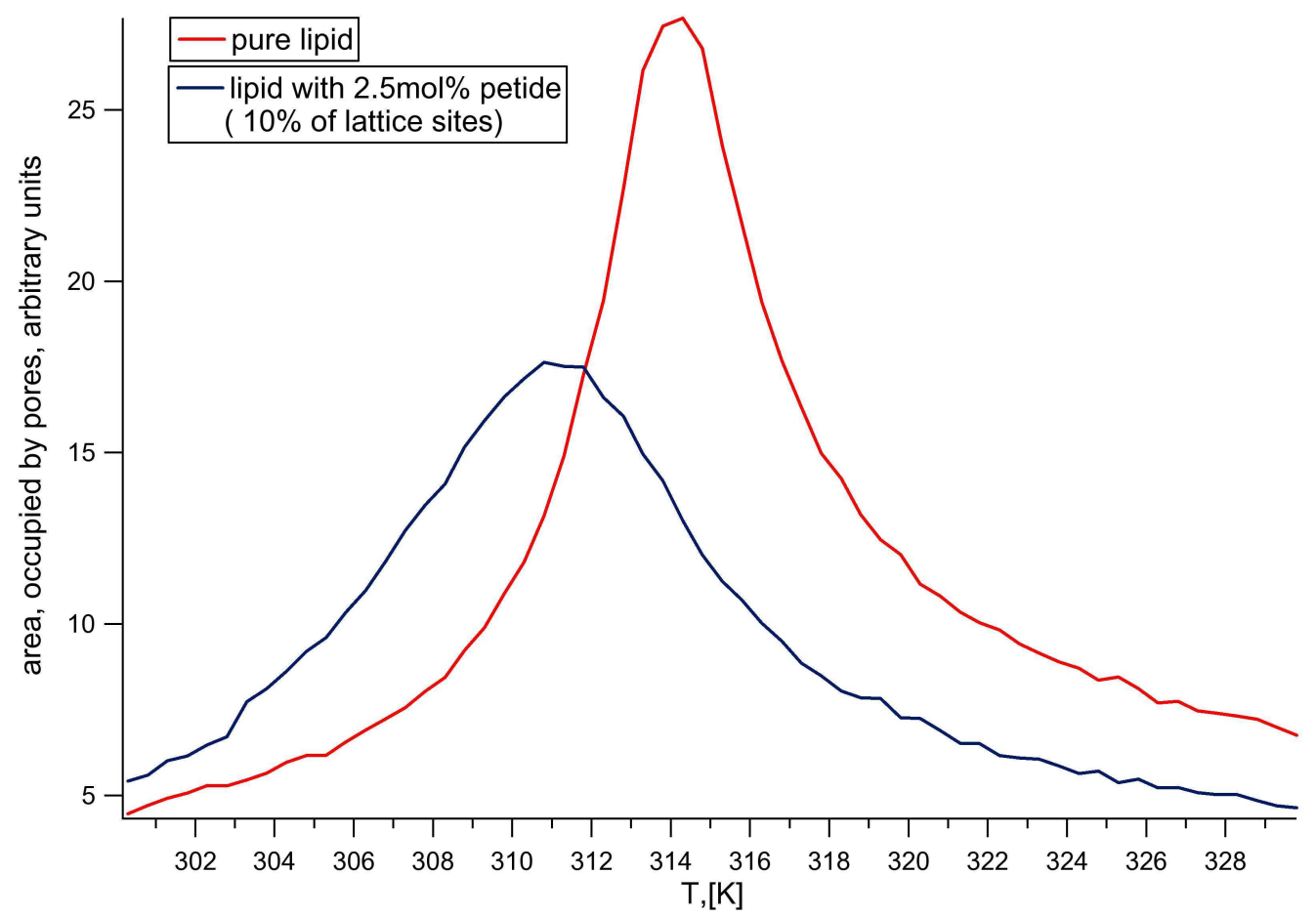

Figure 4.8: Simulation of permeability dependence on temperature for pure lipid membrane, and for membrane with addition of $2.5 \mathrm{~mol} \%$ ( $10 \%$ of lattice sites ) of peptide. Simulation parametrs : $\omega_{l g}=2969 \mathrm{~J} / \mathrm{mol}, \omega_{l f}=522 \mathrm{~J} / \mathrm{mol}, \omega_{l p}=3764$ $J / m o l$. 
However, the theoretical curve is slightly brighter as the experimental one. This can be explained by the overestimation of the interaction energy between the pore and its nearest neighbors.

In the case of a peptide containing membrane ( fig. 4.8 ), we need another parameter $\omega_{l p}$ which describes the interactions between pores and peptides. In our simulation $\omega_{l p}=3764 \mathrm{~J} / \mathrm{mol}$. The parameters which describe the interactions between a peptide and a lipid in the gel state and between a peptide and a lipid in the fluid state are taken from Gramicidin A aggregation studies ( see section 4.2 ). Because of our choice of the $\omega_{l p}$ parameter the addition of the peptide shifts the permeability maximum to lower temperatures, in agreement with the experiment ( see experimental data fig.4.5).

However, we should mention that the permeation rates are more influenced by domain formation behavior ( fluctuations at domain border ) and peptide aggregation behavior ( fluctuations near peptide clusters ) rather than the exact values of $\omega_{l n}$ interactions parameters. $(n=g, f, p)$

Fig. 4.9 shows Monte-Carlo snapshots for a pure lipid membrane (right column), and for a membrane with $2.5 \mathrm{~mol} \%$ ( $10 \%$ of lattice sites ) of peptide( left column) in the phase transition regime and above and below the transition temperature. Domains of gel ( solid-ordered ) and fluid ( liquid disordered ) lipids are presented by dark blue and bright blue correspondingly. Peptides are presented being red. Pores are presented by a white color. One can see that pores tend to appear at domain borders and near peptides.

This supports our assumption about strong local lipid fluctuations near peptides and a strong change of elastic properties of lipid membrane near peptides. 

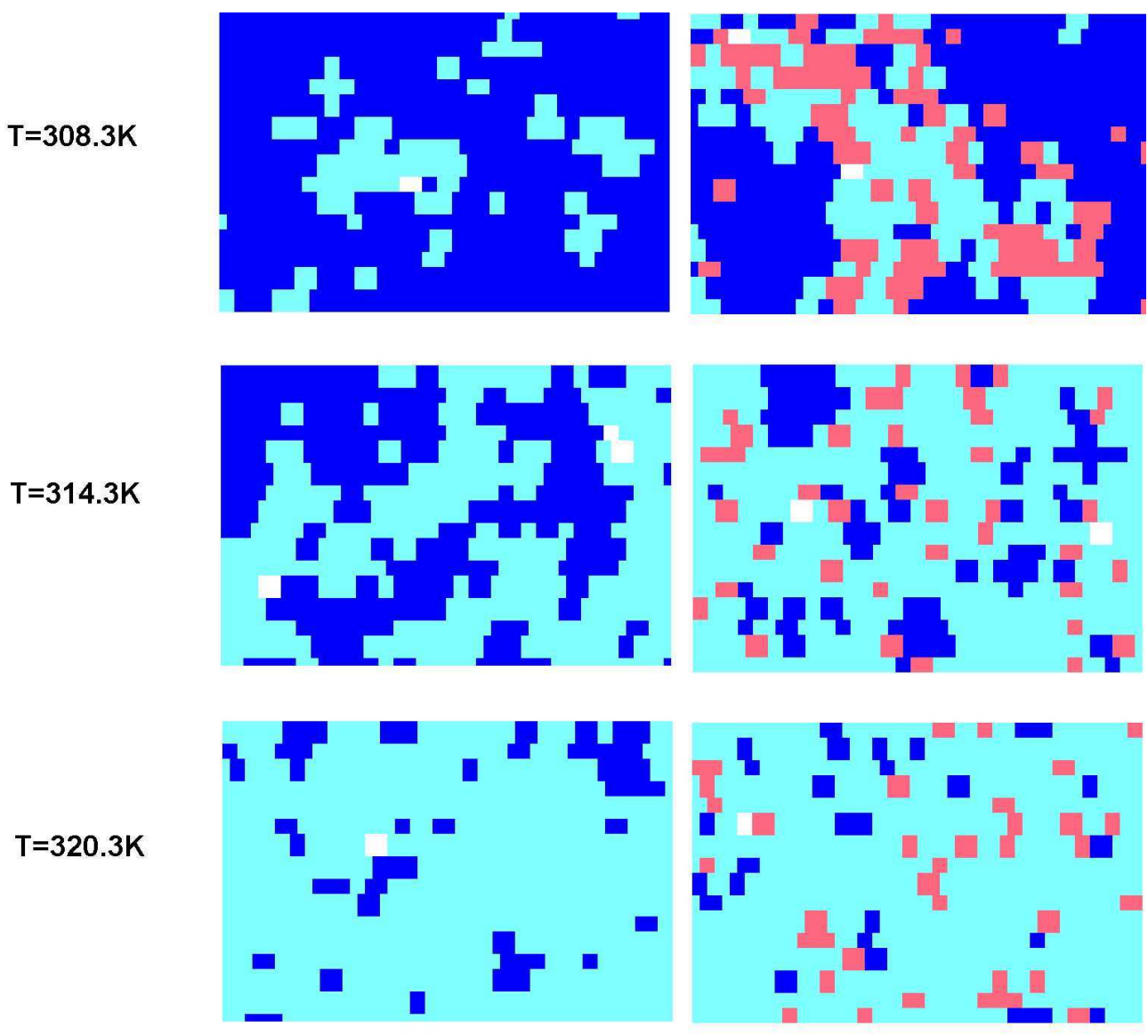

Figure 4.9: Monte-Carlo snapshots for a pure lipid membrane (right column), and for membrane containing 2.5 mol \% ( $10 \%$ of lattice sites ) of peptide( left column) for three different temperatures : below and above the phase transition point and in transition regime. Domains of gel ( solid-ordered) and fluid (liquid-disordered) are presented by dark blue and bright blue colors. Peptides are presented by a red color. Pores are presented by a white color. One can see that the pores tend to appear on domain borders and near peptides. ( here small areas of the simulation matrix are shown with aim to make the pores visible. The simulations was done on the matrix $200 \times 100$. ) 


\subsection{Discussion and perspectives}

In this work it was shown which effect peptides and peptide aggregates have on membrane permeability in terms of fluctuations in the local lipid environment. This effect was observed in experiment, theoretical model and simulation approach were created.

This issue is biologically relevant because such phase transitions occur as well in real cell membranes, i.e. in E.coli bacteria (Pollakowski, 2003), Lung surfactant (Ebel et al., 2001) . In the case of E.coli this transition is located slightly below the temperature they grow at ( fig. 4.10 ). The shift of phase

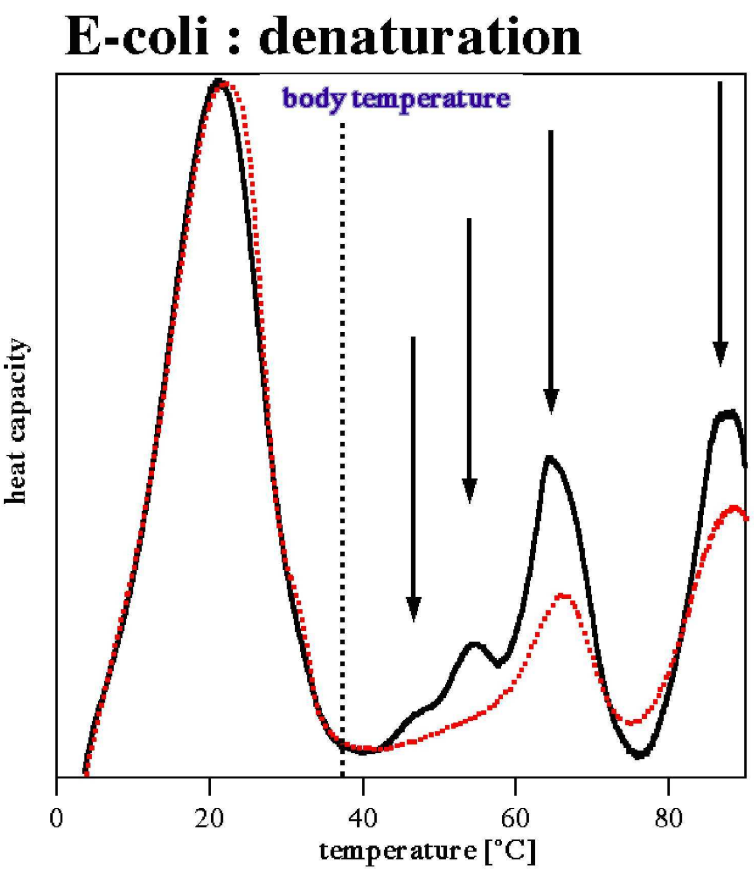

Figure 4.10: Heat capacity profile of E.coli denaturation. The main phase transition is located slightly below body temperature. Picture obtained from T.Heimburg, D. Pollakowski

transition temperature, similar to the one caused by addition of peptides, 
can be caused by other factors, like pressure or $\mathrm{pH}$ changes. Such changes of membrane permeability caused for example because of local $\mathrm{pH}$ changes might be used by nature for regulation of biological function.

From previous studies (Grabitz et al., 2002) follows that, for the lipid membrane a relaxation time, associated with the domain formation processes is proportional to the heat capacity. Theoretically this relaxation time is obtained by an autocorrelation of the enthalpy fluctuations trace, obtained from MC simulations ( fig. 4.11, a) ). A pore opening time can be obtained by an autocorrelation of the trace of the fluctuations in the area, occupied by pores ( fig. 4.11, d) ). Here all autocorrelation operations are done over MC time. Arbitrary units here means that this MC time is related to MC process, have meaning of sequence of states and is not related to real time, and can not be expressed in units of real time, like seconds. From our simulation follows, that the calculated pore opening time ( fig. $4.11 \mathrm{e}$ ), f) ) is proportional to the relaxation time ( fig. $4.11 \mathrm{~b}$ ) to e) calculated for the temperature $308 \mathrm{~K}$ below the phase transition temperature and c) to f) calculated for the temperature $314 \mathrm{~K}$ at the phase transition regime ) in the system, and therefore to the heat capacity. However curve on ( fig. 4.11, f) ) contains some irregularities, which cannot be explained at the moment and is the subject of future investigations.

From our consideration follows, that the permeability in the phase transition regime is higher not only because there are more pores, but also because the pore stays open longer.

Similar dependence was observed experimentally by (Kaufmann and Silman, 1983) by patch-clamp experiments on the lipid membrane containing acetylcholinesterase. In these experiments shift into the transition regime was 


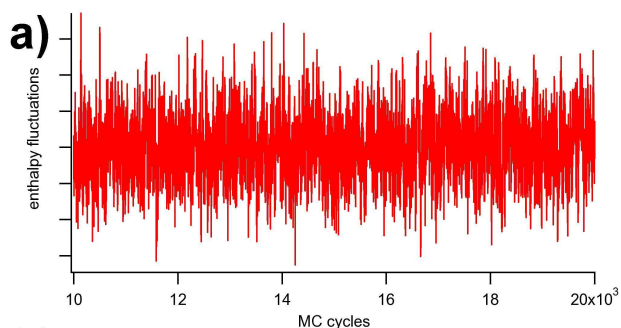

b)

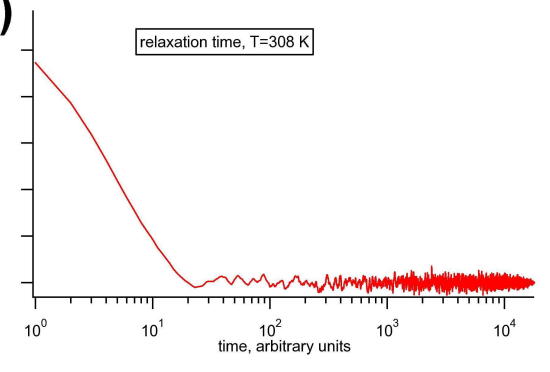

c)

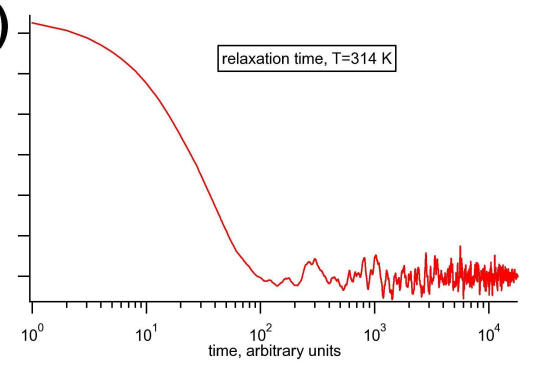

d)

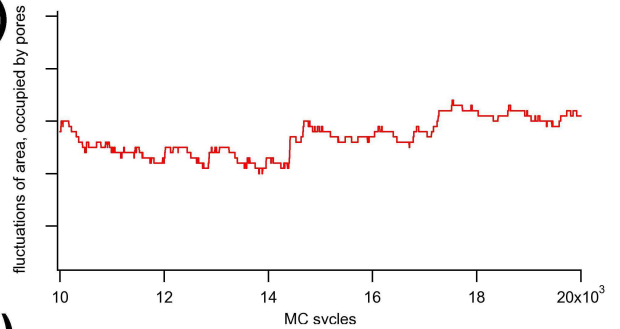

e)

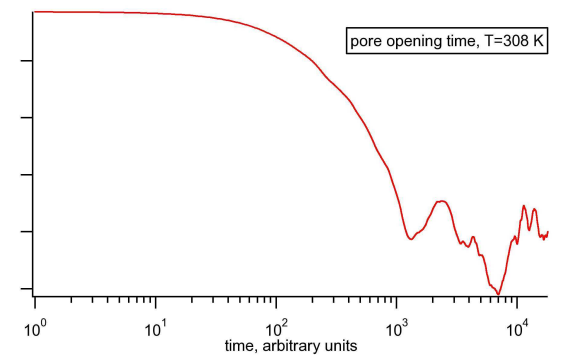

f)

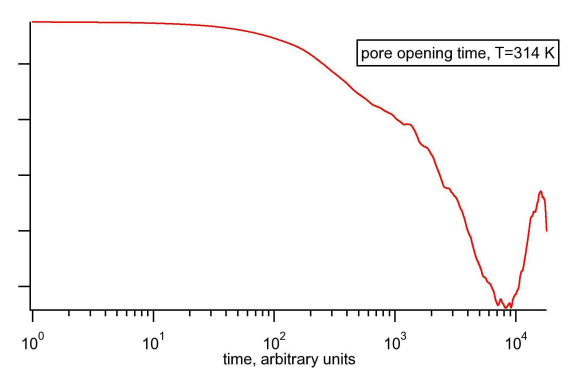

Figure 4.11: a) shows heat capacity fluctuations trace. b) shows relaxation time for $T=308 \mathrm{~K}$ below the phase transition temperature, obtained by the autocorrelation of the heat capacity fluctuations trace. c) shows the relaxation time for $T=314 \mathrm{~K}$ at phase transition temperature. d) shows the fluctuations in area, occupied by pores. e) shows pore opening time for $T=308 \mathrm{~K}$, obtained by autocorrelation of trace of fluctuations in area, occupied by pores. e) shows the pore opening time for $T=314$ $K$. Here all autocorrelation operations are done over $M C$ time. Arbitrary units mean that this $M C$ time is related to the $M C$ process, have the meaning of a sequence of states and it is not related to real time, and therefore can not be expressed in units of real time, like seconds. Obtained by $M C$ simulation pore opening time is proportional to the relaxation time in the system (b) to e) calculated for the temperature $308 \mathrm{~K}$ below the phase transition temperature and c) to f) calculated for the temperature $314 K$ at the phase transition regime).

obtained by change of $\mathrm{pH}$. 
These considerations can give one an idea of a patch-clamp experiment on the lipid membrane, containing a peptide similar to Gramicidin A, done at two or more different temperatures, one of which should be equal to the phase transition temperature.

The main advantage of the used Monte Carlo model in comparison with mean-field models, described in first chapter, is that it takes into account the key features of the membrane complexity : the domain structure of the membrane, inhomogeneity of the peptide distribution.

However, this is a two state model and does not include all the complexity of possible lipid chain configurations at interfaces, whatever gel-fluid, lipid-peptide of pore edge. These border interactions are hidden in the $\omega_{n n}$ interactions parameters. But some assumptions about the pore edge can be made and appearing pores can be considered as hydrophilic or hydrophobic depending on $\omega_{l g}$ and $\omega_{l f}$ parameters ( fig. 4.12). The used values of $\omega_{l g}$
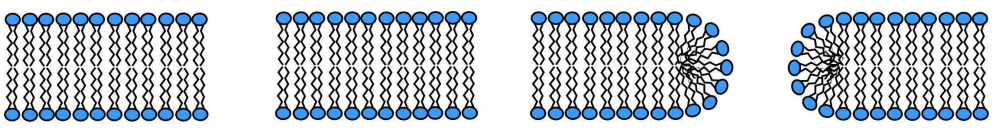

\section{a)}

b)

Figure 4.12: Hydrophobic a) and hydrophylic b) pores.

and $\omega_{l f}$ describes more likely hydrophilic pores.

Used lattice model does not describe the spatial disorder of the in the fluid state. This disorder can be described by slightly modified model - for lipid in fluid phase 4-6 neighbors for interaction can be taken randomly from first coordination sphere. However, one should be aware that such off-lattice models usually show strong hysteresis and therefore are not very well suited 
for the study of critical phenomena.

Our model can be used as well to investigate membrane rupture related problems. At some critical value of parameters small pores diffuse together forming one big pore, which continues to grow, occupying all free space on the lattice - rupture of the membrane occurs ( fig. 4.13 ).

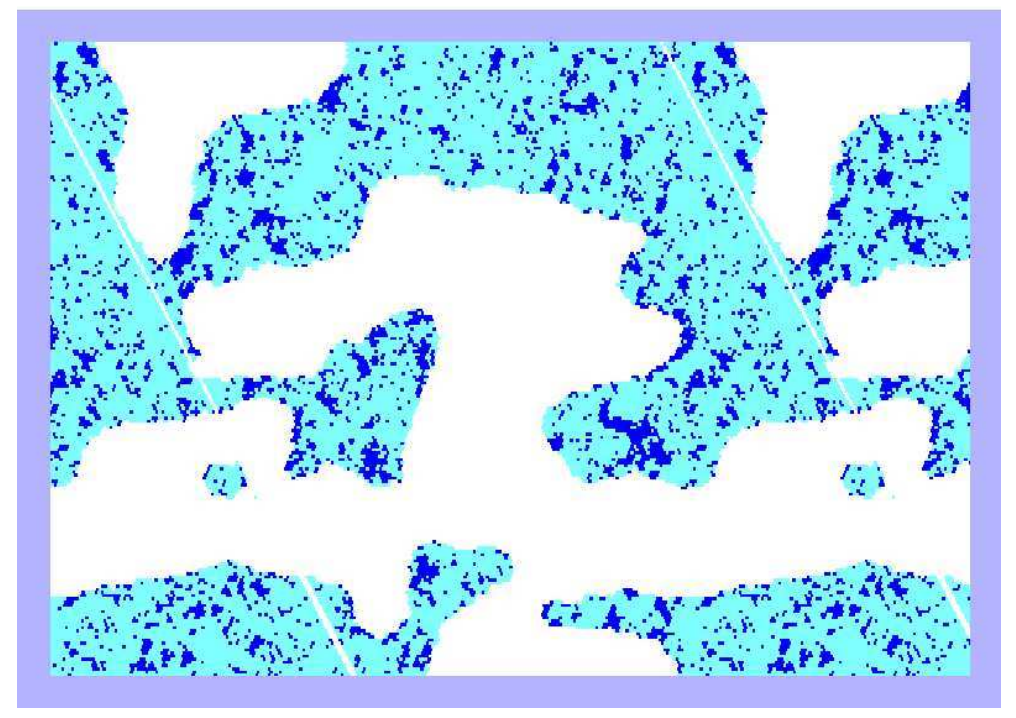

Figure 4.13: Simulation of the rupture of a membrane.

This can be subject of future investigations. Because of the use of a matrix of changeable size our model has an advantage over a model, proposed by Fournier (Founier and Joos, 2003), which uses a matrix of fixed size and several lipids are put to the same lattice point, which leads to a distortion of the membrane topology and an overestimation of the interaction energy.

With a slight modification our model can be also applied to protein insertion problems. Protein absorption kinetics at different temperatures can be studied, and titration calorimetry can provide experimental data for such investigations. This also could also be subject of future research projects. 
Our model can be slightly modified to describe the anisotropy of peptide-lipid interactions and pore formation by pore-forming peptides.

The calorimetry studies of Gramicidin A aggregation revealed interesting structures of the Cp profiles ( fig. 4.14 ). At the phase transition point a

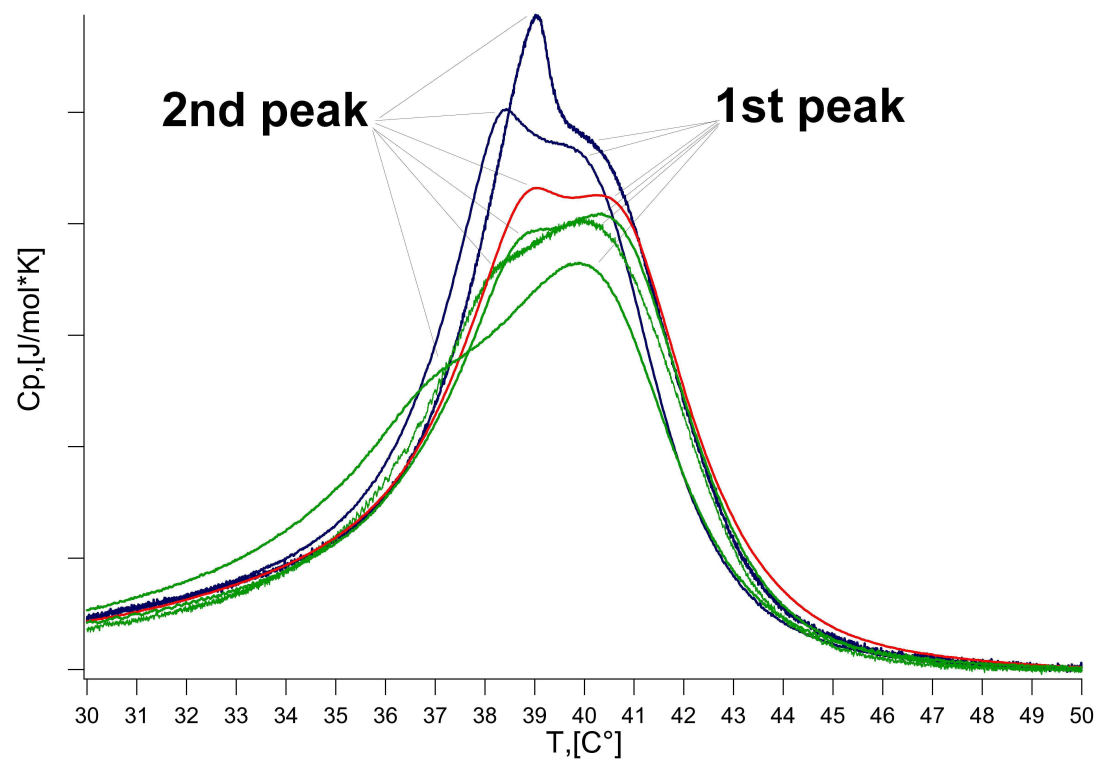

Figure 4.14: Two peak structure of maximum of the experimental heat capacity profile for Gramicidin A. Different curves represent results of the measurements with slightly different peptide concentrations around 2.5 mol\%.

2 - peak structure can be seen. In the shown curves concentration the of Gramicidin A varies slightly around $2.5 \mathrm{~mol} \%$. In the three green curves peak 1 is higher than peak 2, in red curve peaks 1 and 2 have almost the same hight, peak 2 is slightly higher, and in the two blue curves peak 2 is higher athan peak 1. $2.5 \mathrm{~mol} \%$ seems to be critical concentration for this effect, because no similar effect was observed for other Gramicidin A concentrations - $1.25 \mathrm{~mol} \%, 5 \mathrm{~mol} \%$. There is no trivial explanation for this fact. However, with AFM studies of lipid / Gramicidin A systems line shaped aggregates of peptide were found. Such aggregates are not predicted 
by the model, but one can speculate that the appearance or disappearance of such aggregates can change the characteristic length in the system and therefore slightly changes the temperature of the phase transition. If ripple phase formation can be understood as the change of the characteristic length of the system ( characteristic length in ripple phase can be related to the width of ripple stripe and supposed to be infinite in pure gel state) and such big change cause appearance of small peak several degrees under main transition temperature then small change in the characteristic length can cause appearance of second peak only slightly shifted down the temperature, assuming such aggregation have strong influence on the cooperativity. This question is open for further discussions.

The developed FCS method of the measurement of membrane permeability can be applied to a wide range of problems. Membranes with different lipid - peptide compositions can be investigated, chemical composition of solution inside or outside the vesicles can be changed. Dye molecules of different size can be used to study permeability related to fluctuations, or permeability caused by pore-making peptides like melittin, alameticin. Molecules of interest can be labeled, and cross-correlation or FRET techniques can be used to study specific reactions. The use of quenching based methods is also possible. This flexibility of the developed experimental approach open huge perspectives in future research, and particularly can be applied to drug delivery problems. 


\section{Appendix A}

\section{Chromatography procedure}

Sephadex G 75 gel was used to separate lipid vesicles and free Rhodamin 6G dye particles.

At the beginning $5 \mathrm{~g}$ of Sephadex G 75 gel was dissolved in $80 \mathrm{ml}$ water.

Then a small amount of glass wool was put into a fixed vertically glass pipette to make a chromatography column.

After that this column was washed with water.

The next step was the filling of the column with Sephadex gel.

Then ones more the column was washed with water.

The solution, containing vesicles and dye particles was put into the column.

The vesicles were collected in several Eppendorf containers, which were placed under the column. This process was visually controlled.

Then the solution, containing only vesicles was used for the FCS experiments.

Each column should be used only ones.

The gel solution should be stored in the fridge. 


\section{Bibliography}

Andersen, O., H. Appel, E. Bamberg, B. D.D., R. Koeppe 2nd, F. Sigworth, G. Szabo, D. Urry, and A. Wooley (1999). Nat. Struct. Biol. 6, 609.

Branton, D. (1966). Proc. Natl. Acad. Sci. USA 55, 1048.

Calhoun, W. and G. Shipley (1979). Biochim. Biophys. Acta 555, 436-441.

Cruzeiro-Hansson, L. and O. Mouritsen (1988). Biochim. Biophys. Acta 944 , 63-72.

Danielli, J. and D. J. (1935). J.Cell.Comp.Physiol. 5, 495-508.

Doniach, S. (1978). J. Phys. Chem. 68, 4912-4916.

Ebel, H., P. Grabitz, and T. Heimburg (2001). J. Phys. Chem. B 105, 73537360.

Eigen, M. and R. R. (1994). Proc. Natl. Acad. Sci. USA 91, 5740-5747.

Evans, E. and D. Needham (1987). J. Phys. Chem. 91, 4219-4228.

Finean, J. and R. Burge (1963). J. Mol. Biol. 7, 672.

Founier, L. and B. Joos (2003). Phys. Rev. E 67, 051908-1.

Gennis, R. (1989). Biomembranes. In molecular structure and function. Springer Verlag, New York. 
Glauber, R. (1963). Journal of Mathematical Physics 4, 294-307.

Grabitz, P., V. Ivanova, and T. Heimburg (2002). Biophys. J. 82, 299-309.

Groot, R. and K. Rabone (2001). Biophys. J. 81, 725-736.

Hamburger, H. (1887). Arch. Anat. Physiol 0, 31-47.

Hamilton, R. and E. Kaler (1990). J. Phys. Chem. 94, 2560-2566.

Heimburg, T. (1998). Biochim. Biophys. Acta 1415, 147-162.

Heimburg, T. (2000). Curr. Opin. Colloid interface Sci. 5, 224-231.

Heimburg, T. and R. Biltonen (1996). Biophys. J. 70, 84.

Hill, T. (1960). An introduction to statistical Thermodynamics. Dover New York.

Ivanova, V., I. Makarov, T. Schäffer, and T. Heimburg (2003). Biophys. J. 84, 2427-2439.

Kaufmann, K. and I. Silman (1983). Biophys. Chem. 18, 89-99.

Ketchem, R., W. Hu, and T. Cross (1993). Science 261, 1457-1460.

Kleinzeller, A. (1999). Current Topics in Membranes 48, 1-22.

Kwok, R. and E. Evans (1981). Biophys. J. 35, 637-652.

Landbrooke, B. and D. Chapman (1969). Chem.Phys.Lipids 3, 304-367.

Landbrooke, B., R. Williams, and D. Chapman (1968). Biochim. Biophys. Acta 150, 333-340.

Leontiadu, H., A. Mark, and S. Marrink (2004). Biophys. J. 86, 2156-2164. 
Luzzati, V. (1968). In Biological Membranes. Academic Press New York.

McIntosh, T. (1999). Current Topics in Membranes 48, 23-49.

Meyer, H. (1899). Arch.Exp.Pathol.Pharmacol. 42, 109-118.

Mouritsen, O. and M. Bloom (1984). Biophys. J. 46, 141-153.

Nägeli, C. and C. Cramer (1855). Pflanzenphysiologische Untersuchungen. Shulthess Zürich.

Nagle, J. and H. Scott (1978). Biochim. Biophys. Acta 513, 236-243.

Needham, D. and D. Zhelev (1996). In Vesicles (M.Rosoff ed.). Dekker New York.

Newson, W. (1773). Philos. Trans. R. Soc. London 63, 306-324.

Nielsen, K., T. Bjornholm, and O. Mouritsen (2000). Nature 404, 532.

Overton, E. (1855). Studien über die Narkoze, zugleich ein Beitrag zur allgemainen Pharmakologie. Gustav Fisher Jena.

Overton, E. (1895). Vieteljahrsschr. Naturforsch. Ges. Zürich 40, 159-201.

Overton, E. (1899). Vieteljahrsschr. Naturforsch. Ges. Zürich 44, 88-114.

Papahadjopoulos, D., K. Jacobsen, S. Nir, and T. Isac (1973). Biohim. Biophys. Acta 311, 330-348.

Parsegian, A. (1969). Nature 221, 844-846.

Paula, S. and D. Deamer (1999). Current Topics in Membranes 48, 78-95.

Pfeffer, W. (1877). Osmotische Untersuchungen. W. Engelmann Zuerich. 
Pfeffer, W. (1886). Unters.a.d.Bot.Inst.Tübingen 2, 179-331.

Plotnikov, V., J. Brandts, L. L.N., and B. J.F. (1997). Analytical biochemistry 250, 237-244.

Pollakowski, D. (2003). Termodinamishe und strukturelle untersuchungen an künstlichen und biologischen Membranen grundlegende Eigenshaften und der Einfluss kleiner Moleküle. Diplomarbait, Goettingen.

Quincke, G. (1888). Ann.Phys.(Leipzig) 35, 580-642.

Ramsden, W. (1904). Z.Phys.Chem. 47, 336-346.

Rigler, R. and E. Elson (2001). Fluorecence Correlation Spectroscopy Theory and Applications. Springer.

Robertson, J. (1957). J.Physiol.London 140, 58-59.

Schmitt, F. and K. Palmer (1940). Cold Spring Harbor Symp. Quant. Biol. 8, $94-101$.

Shillcock, J. and U. Seifert (1988). Biophys. J. 74, 1754-1766.

Singer, S. and G. Nicolson (1972). Science 175, 720-731.

Vries de, H. (1871). Arch.Neerl.Sci.Exactes Nat. 6, 117-126.

Vries de, H. (1884). Jahrb.Wiss.Bot. 14, 429-601. 


\title{
LEBENSLAUF
}

\section{PERSÖNLICHE DATEN}

\author{
Makarov Ivan \\ Geburtsdatum : 8. Oktober 1975 \\ Geburtsort : Kiew \\ Staatsangehörigkeit : Ukraine \\ E-mail: imakaro@gwdg.de
}

\section{HOCHSCHULAUSBILDUNG}

1.9.1992-1.9.1994 Moskau Institut für Physik und Technik, Fakultät für allgemeine und angewandte Physik

1.9.1994-30.6.1999 Kiew Taras Chevchenko Universität, Physikfakultät, Lehrstuhl für Festkörperoptik

1999 Diplom in Physik (Vertiefung : Festkörperphysik) Thema der Diplomarbeit : Optischeleitfähigkeitsberechnung von ungeordneten Metalllegierungen Untersuchungsgebiete : Simulation in der Physik der ungeordneten Materie, optische Eigenschaften von amorphen Legierungen

\section{BERUFSTÄTIGKAIT}

1.1999-9.1999 Wissenschaftler im Zentrum für Leitungs- und Kabelanlagen des Staatskomitees für Fernmeldewesen der Ukraine Untersuchungsgebiete : Forschungen im Fiberoptikgebiet, Solitonübertragungssystem für Femtosekundenbereichentwicklung

15.11.1999-1.9.2001 Wissenschaftler am Institut für Materialforschung der Akademie der Wissenschaften der Ukraine Untersuchungsgebiete : Forschungen im Nanotechnologiegebiet, elektronische Eigenschaften und Supraleitfähigkeit von Nanosystemen

4.2.2002-31.3.2005 Wissenshaftlischer Mitarbeiter am Max Plank Institut für Biophysikalische Chemie bei Prof. Dr. Thomas Heimburg. Untersuchungsgebiete : Forschung in Membranbiophysik und Thermodynamik über theoretische und experimentelle Beschreibung der Permeabilität von Membranen. 